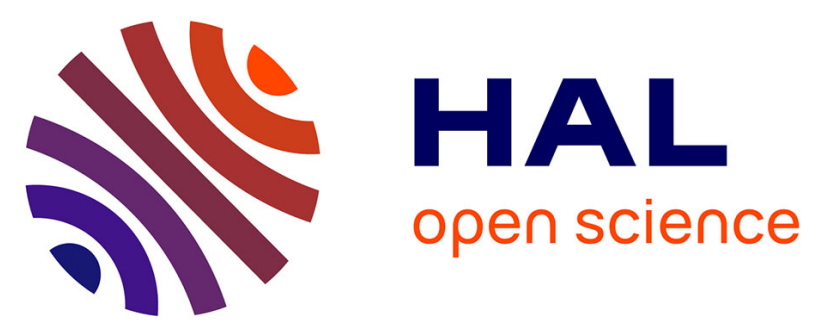

\title{
Nouvelles données sur la chronologie des sites paléolithiques en contexte loessique du Nord-Est et du Sud-Est de la Roumanie (Périphérie orientale des Carpates)
}

Sanda Balescu, Alain Tuffreau, Roxana Dobrescu, Patrick Auguste, Jean-Jacques Bahain, Michel Lamothe, Alexandru Petculescu, Qingfeng Shao

\section{To cite this version:}

Sanda Balescu, Alain Tuffreau, Roxana Dobrescu, Patrick Auguste, Jean-Jacques Bahain, et al.. Nouvelles données sur la chronologie des sites paléolithiques en contexte lœessique du Nord-Est et du Sud-Est de la Roumanie (Périphérie orientale des Carpates). L'anthropologie, 2018, 122 (2), pp.87-110. 10.1016/j.anthro.2018.02.001 . hal-02341076

\author{
HAL Id: hal-02341076 \\ https://hal.science/hal-02341076
}

Submitted on 6 Nov 2019

HAL is a multi-disciplinary open access archive for the deposit and dissemination of scientific research documents, whether they are published or not. The documents may come from teaching and research institutions in France or abroad, or from public or private research centers.
L'archive ouverte pluridisciplinaire HAL, est destinée au dépôt et à la diffusion de documents scientifiques de niveau recherche, publiés ou non, émanant des établissements d'enseignement et de recherche français ou étrangers, des laboratoires publics ou privés. 


\section{ScienceDirect}

Article original

\section{Nouvelles données sur la chronologie des sites paléolithiques en contexte lœssique du Nord-Est et du Sud-Est de la Roumanie (Périphérie orientale des Carpates)}

New data on the chronology of Palaeolithic sites in loessic environments of northeastern and southeastern Romania (Eastern periphery of the Carpathian Mountains)

Sanda Balescu ${ }^{\text {a,* }}$, Alain Tuffreau ${ }^{\text {a }}$, Roxana Dobrescu ${ }^{\mathrm{b}}$, Patrick Auguste $^{\mathrm{c}}$, Jean-Jacques Bahain ${ }^{\mathrm{d}}$, Michel Lamothe ${ }^{\mathrm{e}}$, Alexandru Petculescu ${ }^{\mathrm{f}}$, Qingfeng Shao ${ }^{\mathrm{g}}$

${ }^{\text {a } U M R} 8164$ CNRS, Laboratoire Halma, Université de Lille, bâtiment de Géographie, 59655 Villeneuve-d'Ascq Cedex, France

${ }^{\mathrm{b}}$ Institut d'Archéologie "Vasile Pârvan », 11, rue Henri Coandă, 010667 Bucarest, Roumanie

${ }^{\mathrm{c}}$ UMR 8217 CNRS, Laboratoire Géosystèmes, Université de Lille, Bâtiment SN5, 59655 Villeneuve-d'Ascq Cedex, France

${ }^{\mathrm{d}}$ Unité Histoire naturelle de l'Homme Préhistorique (HNHP, UMR7194), Département Homme et Environnement du Muséum national d'Histoire naturelle, 1, rue René-Panhard, 75013 Paris, France

${ }^{\mathrm{e}}$ Université du Québec à Montréal, Département des Sciences de la Terre et de l'Atmosphère, Laboratoire de luminescence LUX, H3C 3P8 Montréal, Canada

f “Emil Racovita” Institute of Speleology, 11, rue Frumoasa, R-010985 Bucarest 12, Roumanie

${ }^{\mathrm{g}}$ College of Geography Science, Nanjing Normal University, Nanjing 210023, Chine

\section{Résumé}

Dans le cadre de cette étude, la chronologie de plusieurs gisements intralœssiques du Paléolithique supérieur et moyen, situés en Moldavie, en Dobrogea et dans la plaine orientale du Danube, a été réexaminée

\footnotetext{
* Auteur correspondant.

Adresse e-mail : sanda.balescu@univ-lille1.fr (S. Balescu).
} 
et précisée en s'appuyant sur de nouvelles données chronologiques (dates IRSL sur lœss et dates ESR/U-Th sur dents) ainsi que sur des arguments biostratigraphiques et pédostratigraphiques. Des témoins d'occupation du Paléolithique moyen ont été mis au jour dans le lœss L2 du SIM 6 (épisode interstadiaire), le pédocomplexe S2 du SIM 7 et le pédocomplexe de sols rougeâtres S3 vraisemblablement attribué au SIM 9. Ces résultats nous amènent à reconsidérer l'étendue chronologique du Paléolithique moyen de Roumanie qui jusqu'au début des années 2000 (Păunescu, 1999a, b ; Cârciumaru, 1999) était limitée au Pléistocène supérieur (chronologie courte : 130-35 ka ; SIM 5-SIM 3). Nous démontrons donc ici la présence de traces d'occupation humaine dès le Pléistocène moyen à la périphérie orientale des Carpates roumaines, le long de la vallée du Danube et de son affluent le Prut.

(C) 2018 Elsevier Masson SAS. Tous droits réservés.

Mots clés : Paléolithique ; Lœss ; Datation IRSL ; Datation ESR/U-Th ; Roumanie

\begin{abstract}
In the present study, the chronology of several intraloessic Upper and Middle Palaeolithic sites, located in Moldova, Dobrogea and the eastern Danube Plain, is re-examined and better defined on the basis of new chronological data (IRSL ages on loess, ESR/U-Th ages on teeth) and also biostratigraphic and pedostratigraphic evidence. Middle Palaeolithic occupations have been identified in the MIS 6 loess L2 (interstadial episode), the MIS 7 pedocomplex S2 and the red soils S3 presumably assigned to MIS 9. These results are questioning the chronological extension of the Middle Palaeolithic in Romania which until early 2000 (Păunescu, 1999 a, b; Cârciumaru, 1999) was restricted to the Upper Pleistocene (short chronology: 130-35 ka; MIS 5-MIS 3). Hence, we demonstrate herein that Humans were already present during the Middle Pleistocene at the eastern periphery of the Carpathian Mountains, along the Danube valley and its tributary, the Prut River.
\end{abstract}

(C) 2018 Elsevier Masson SAS. All rights reserved.

Keywords: Paleolithic; Loess; IRSL Dating; ESR/U-Th dating; Romania

\title{
1. Introduction
}

Les lœss roumains, situés à la périphérie orientale des Carpates (Fig. 1), s’inscrivent dans la continuité des lœss de Bulgarie, d’Ukraine et de la République Moldave. Les séquences lœssiques du SE de la Roumanie sont exceptionnelles et uniques par leur épaisseur (plus de 20$25 \mathrm{~m}$ ) et leur extension chronologique (Balescu et al., 2003 ; Radan, 2012). La séquence lœssique de Tuzla, en particulier, située en Dobrogea, en bordure de la Mer Noire (Fig. 1), y est la plus représentative (Balescu et al., 2003). Elle atteint 20 m d'épaisseur et remonte à la limite BruhnesMatuyama (B/M) soit environ 800 ka. Elle est caractérisée par une superposition de sept unités lœssiques désignées L1 à L7 par Panaiotu et al. (2001) et six paléosols interstratifiés désignés S1 à S7, du haut vers le bas (Fig. 2). La limite B/M a été identifiée dans le lœss L7 (Balescu et al., 2003). Les paléosols S3 à S7 sont des sols rougeâtres rubéfiés de type méditerranéen qui attestent d'un environnement chaud et humide. Les paléosols S1 et S2 sont des sols forestiers brunâtres caractéristiques d'environnements plus arides (Panaiotu et al., 2001).

Le premier cadre chronostratigraphique des lœss roumains fut établi par A. Conea (1969). En l'absence d'argument chronologique, il reposait uniquement sur des arguments pédostratigraphiques (Fig. 3). Le premier paléosol rougeâtre (S3) à partir de la surface, était rapporté au 


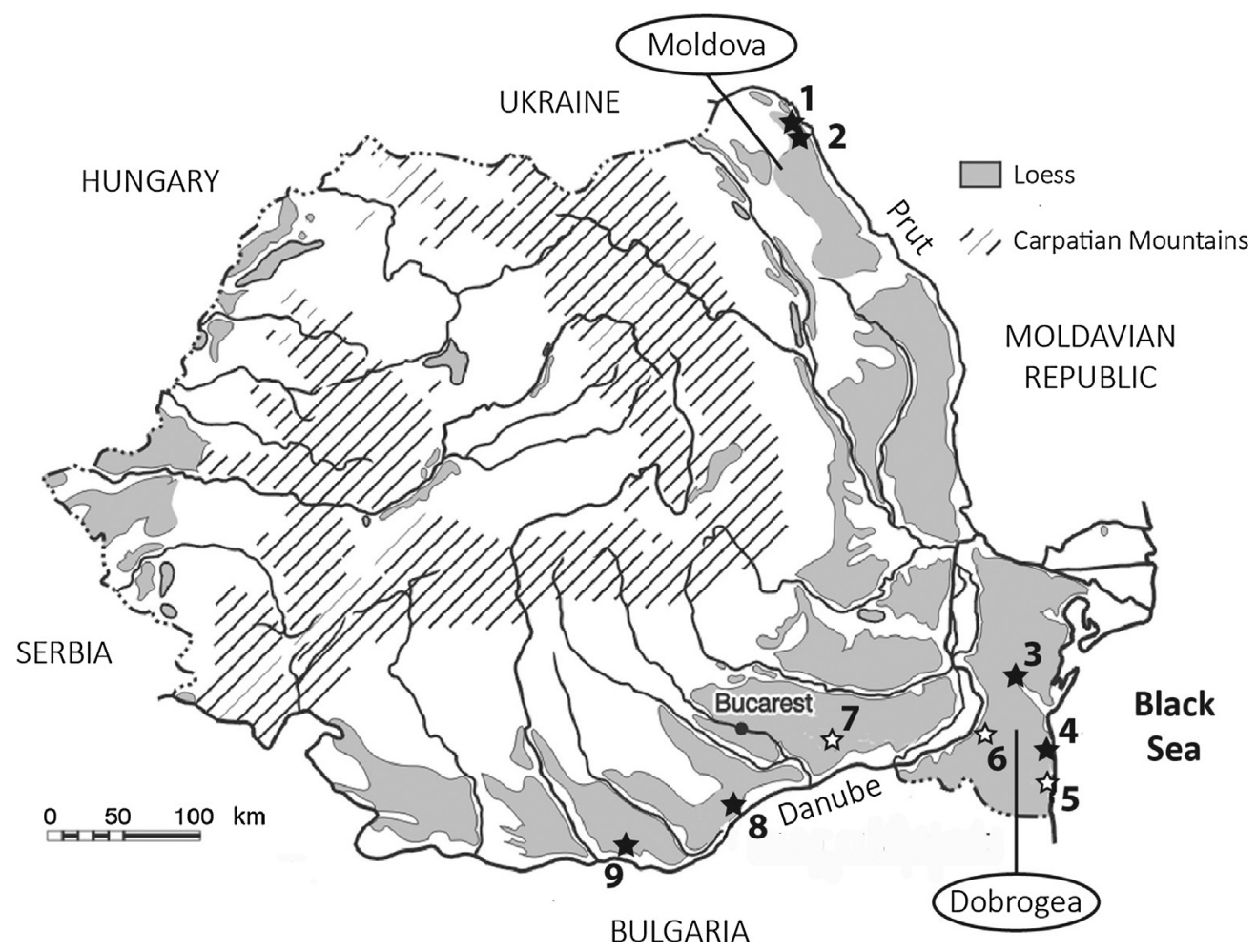

Fig. 1. Carte de localisation des gisements roumains étudiés. Sites paléolithiques étudiés (étoile noire) et séquences lœssiques de référence (étoile blanche).

Location map of the studied Romanian sites. Studied Paleolithic sites (black stars) and reference loess sequences (white stars).

Dernier Interglaciaire (ou stade isotopique marin SIM 5). Les trois lœss surmontant le paléosol S3 (L1, L2, L3) étaient par conséquent attribués au Dernier Glaciaire (SIM 4-2). Les paléosols bruns, S2 et S1, sus-jacents à S3, étaient ainsi rapportés à des épisodes interstadiaires de la Dernière Glaciation.

Cette chronologie courte a cependant été remise en question par les premiers résultats de la datation par luminescence des lœss roumains (Balescu et al., 2003) qui ont démontré que ces derniers s'inscrivent plutôt dans une chronologie longue (Fig. 3). L'application de la méthode de datation par luminescence IRSL (Infrared Stimulated Luminescence) aux grains de feldspaths potassiques (K) des lœss de Tuzla a en effet permis de montrer que les lœss L1, L2 et L3, se sont mis en place au cours des trois dernières glaciations correspondant respectivement aux SIM 2-4, 6 et 8 (Fig. 2 et 3). Le premier paléosol brunâtre (S1) sous la surface, est dès lors attribué au Dernier Interglaciaire (SIM 5) et le premier paléosol rougeâtre (S3) par contre correspond au SIM 9.

Ces conclusions ont été ultérieurement confortées par les résultats de la datation par luminescence de deux autres séquences lœssiques :

- celle de Mostiştea dans la plaine orientale du Danube ;

- celle de Mircea Vodă en Dobrogea (Fig. 1). 

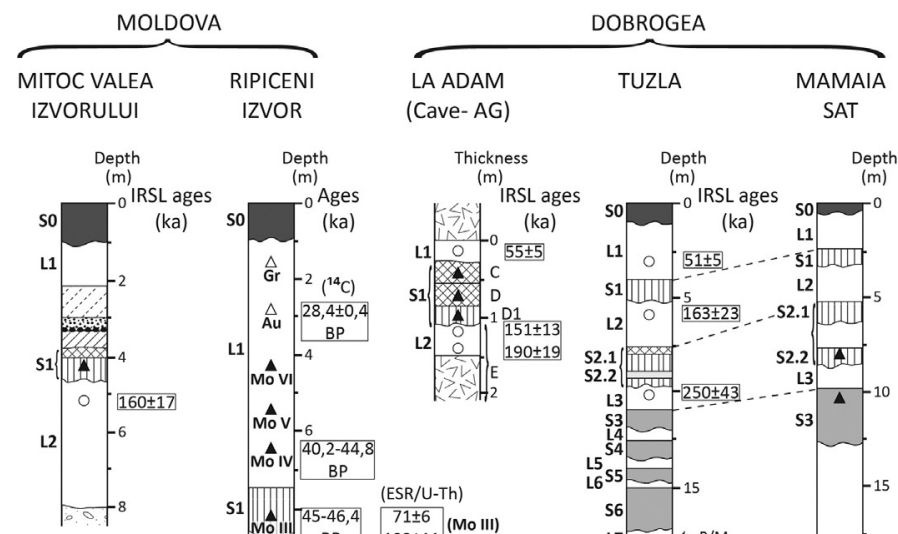

$$
\text { (Cave- AG) }
$$$$
\begin{gathered}
\text { Depth } \\
(\mathrm{m})
\end{gathered}
$$
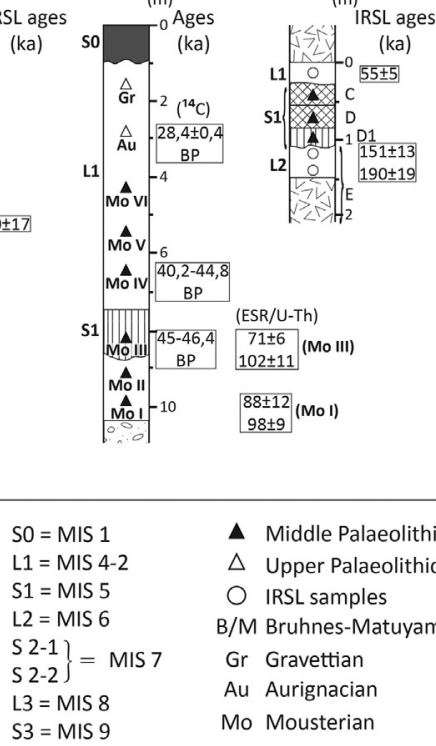

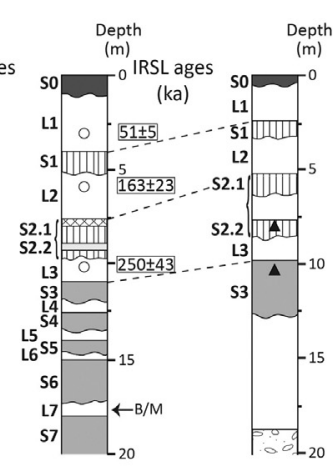

hickness

IRSL age
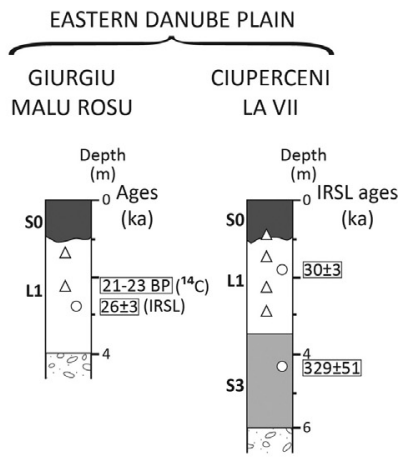

Fig. 2. Lithostratigraphie, données chronologiques et interprétation chronostratigraphique des séquences lœssiques du sud-est et du nord-est de la Roumanie : séquences associées aux gisements paléolithiques et séquence lossique de référence de Tuzla.

Lithostratigraphy, chronological data and chronostratigraphic interpretation of the loessic sequences from southeastern and northeastern Romania: sequences associated with the Palaeolithic sites and the reference loess sequence of Tuzla.

Trois techniques différentes de datation par luminescence leur ont été appliquées :

- IRSL sur feldspaths potassiques (Balescu et al., 2010) ;

- OSL sur quartz (Timar et al., 2010 ; Timar-Gabor et al., 2011) ;

- post-IR IRSL sur grains fins (Vasiliniuc et al., 2012).

Les lœss du NE et du SE de la Roumanie renferment de nombreux niveaux d'industries lithiques du Paléolithique supérieur (12 000-35 000 ans BP non calibrées) et moyen (> 35000 ans BP). Jusqu'au début des années 2000, la chronologie des gisements de plein air, en contexte lœssique, attribués au Paléolithique supérieur et moyen (Păunescu, 1999a, 1999b ; Cârciumaru, 1999) s'appuyait sur la chronologie courte des séquences lœssiques établie par A. Conea (1969) et sur la méthode ${ }^{14} \mathrm{C}$ conventionnelle. L'étendue du Paléolithique moyen de Roumanie était alors restreinte au dernier cycle interglaciaire-glaciaire (SIM 5-SIM 3 ; 130-35 ka) (Păunescu, 1999a, 1999b ; Cârciumaru, 1999) ce qui contraste avec le Paléolithique moyen d'Europe occidentale et centrale qui est apparu il y a environ 300 ka (Tuffreau, 1979) (Fig. 3). Dans les années 1960-1980, de nombreuses dates ${ }^{14} \mathrm{C}$ conventionnelles de 40-45 ka ont en effet été utilisées comme argument 


\begin{tabular}{|c|c|c|c|c|c|c|}
\hline \multicolumn{3}{|c|}{ Isotopic chronology } & \multicolumn{2}{|c|}{ Chrono-cultural framework } & \multicolumn{2}{|c|}{$\begin{array}{l}\text { Loessic sequences of } \\
\text { Eastern Romania }\end{array}$} \\
\hline \multirow[b]{2}{*}{ MIS } & \multirow{2}{*}{$\begin{array}{c}\text { Time } \\
\text { (ka) }\end{array}$} & Benthic $\delta^{18} 0$ (ppm) & \multirow{2}{*}{$\begin{array}{l}\text { NW Europe } \\
\text { (Tuffreau, 1979) }\end{array}$} & \multirow{2}{*}{$\begin{array}{c}\text { Romania } \\
\text { (Paunescu, 1999; } \\
\text { Carciumaru 1999) }\end{array}$} & \multirow{2}{*}{$\begin{array}{l}\text { Conea } \\
\text { (1960) }\end{array}$} & \multirow{2}{*}{$\begin{array}{l}\text { Balescu et } \\
\text { al (2003) }\end{array}$} \\
\hline & & $5.2, \quad 4, \quad 2.8$ & & & & \\
\hline 1 & \multirow{6}{*}{$\begin{array}{l}- \\
- \\
- \\
- \\
- \\
-\end{array}$} & & & & so & so \\
\hline 2 & & & \multirow{2}{*}{$\begin{array}{c}\text { Upper } \\
\text { Palaeolithic }\end{array}$} & \multirow{2}{*}{$\begin{array}{c}\text { Upper } \\
\text { Palaeolithic }\end{array}$} & L1 & \multirow{4}{*}{$L 1$} \\
\hline \multirow{2}{*}{3} & & & & & S1 & \\
\hline & & & \multirow{9}{*}{$\begin{array}{c}\text { Middle } \\
\text { Palaeolithic }\end{array}$} & \multirow[b]{3}{*}{$\begin{array}{c}\text { Middle } \\
\text { Palaeolithic }\end{array}$} & $\frac{L 2}{S 2}$ & \\
\hline 4 & & & & & 25 & \\
\hline 5 & & & & & S3 & S1 \\
\hline 6 & \multirow{6}{*}{$\begin{array}{ll}- & \\
- & 150 \\
- & \\
- & \\
- & \\
- & 200 \\
- & \\
- & \\
- & \\
- & 250 \\
- & \\
- & \\
- & \\
- & 300 \\
- & \\
- & \end{array}$} & \multirow{6}{*}{$\frac{z}{3}$} & & & & L2 \\
\hline & & & & & & \\
\hline 7 & & & & & & 52 \\
\hline 8 & & & & & & L3 \\
\hline 9 & & & & & & 53 \\
\hline & & & & & & \\
\hline
\end{tabular}

Fig. 3. Extension chronologique du Paléolithique en Roumanie et chronostratigraphie des séquences lossiques de l'Est de la Roumanie ( $\mathrm{L}=$ lœss ; $\mathrm{S}=$ paléosol).

Chronological extension of the Palaeolithic in Romania and chronostratigraphy of the loessic sequences of eastern Romania $(L=$ loess; $S=$ palaeosol $)$.

chronologique en faveur d'une attribution des gisements du Paléolithique moyen de Roumanie au Dernier Glaciaire. Or, ces dates ${ }^{14} \mathrm{C}$ ne constituent que des âges minimum dans la mesure où elles se situent à la limite de la méthode $(\sim 40 \mathrm{ka})$.

La remise en question de la chronologie courte des lœss roumains par les premiers résultats de la datation IRSL (Balescu et al., 2003), nous a amené à revoir l'interprétation chronologique de plusieurs gisements contenant du matériel attribuable au Paléolithique supérieur et moyen fouillés dans les années 1960-1980 et à préciser l'étendue du Paléolithique moyen de Roumanie, en appliquant dès 2004, la méthode IRSL aux dépôts lœssiques associés à ces gisements.

Dans cet article, nous présentons une synthèse des données chronologiques relatives à des gisements paléolithiques intralœssiques qui se répartissent dans les trois régions suivantes (Fig. 1) :

- la Moldavie au NE de la Roumanie, le long de la vallée du Prut ;

- la Dobrogea au SE de la Roumanie près de la Mer Noire ;

- la plaine orientale du Danube. 
Cette synthèse regroupe deux types de gisements paléolithiques :

- d'une part ceux fouillés anciennement, mais encore accessibles qui ont dès lors fait l'objet de nouvelles fouilles, permettant ainsi le prélèvement et la datation IRSL d'échantillons de lœss (Giurgiu Malu Roşu, Mitoc Valea Izvorului, Ciuperceni LaVii, La Adam) ;

- d'autre part, ceux aujourd'hui disparus ou inaccessibles (Mamaia Sat et Ripiceni Izvor) mais, dont le matériel lithique et faunique conservé à l'Institut d'Archéologie « Vasile Pârvan » de Bucarest a été réexaminé, et pour lesquels une nouvelle interprétation chronologique est ici proposée à la lumière de nouveaux arguments (dates ESR/U-Th, données biostratigraphiques et pédostratigraphiques).

\section{Méthodes de datation mises en ouvre}

\subsection{Luminescence (IRSL)}

La méthode de datation par luminescence IRSL (Infrared Stimulated Luminescence ; Hütt et al., 1988) est une forme particulière d'Optically Stimulated Luminescence (OSL) réservée aux feldspaths. Lorsque ces minéraux sont stimulés par des photons du proche-infrarouge $(<880 \mathrm{~nm})$, ils émettent un signal IRSL qui mesure le temps écoulé depuis leur dépôt ou leur dernière exposition à la lumière solaire.

La technique IRSL mise en œuvre est identique à celle qui a été appliquée aux séquences lœssiques de référence du SE de la Roumanie (voir Balescu et al., 2003, 2010). Elle repose sur l'analyse du signal IRSL des feldspaths potassiques grossiers (densité $<2,58 \mathrm{~g} \cdot \mathrm{cm}^{-3}$ ) : 40$60 \mu \mathrm{m}$ ou $60-80 \mu \mathrm{m}$. La paléodose (dose totale, dénommée De, de radiation reçue par le minéral depuis son enfouissement) est estimée par la méthode des doses $\gamma$ additives (MDA) appliquée à des aliquotes multiples : différentes aliquotes multigrains $(20 \mathrm{mg})$ d'un même échantillon sont irradiées à température ambiante au moyen d'une source $\gamma$ de ${ }^{60} \mathrm{Co}$. Les aliquotes sont ensuite préchauffées à $160^{\circ} \mathrm{C}$ pendant 10 heures. Les contributions des rayonnements externes $(\alpha, \beta$ et $\gamma)$ et internes $(\beta)$ à la dose annuelle $(\mathrm{Da})$ sont calculées à partir des teneurs en $\mathrm{U}$, Th et $\mathrm{K}$ du sédiment et des teneurs en $\mathrm{K}$ interne des grains qui ont toutes été mesurées par activation neutronique. La contribution du rayonnement cosmique est calculée en tenant compte des profondeurs actuelles des échantillons. La teneur en eau est estimée à $20 \pm 5 \%$; elle correspond à la moyenne entre la teneur en eau actuelle et la teneur à saturation.

En raison de l'instabilité du signal IRSL des feldspaths («fading ») qui se traduit par une perte spontanée de signal au cours du temps et donc une sous-estimation de l'âge (Huntley et Lamothe, 2001), les âges IRSL mesurés ont été corrigés pour le " fading » en utilisant la procédure corrective de Mejdahl $(1988,1989)$.

\section{2. $E S R / U-T h$}

Lors de la fossilisation, une incorporation d'uranium dans les différents tissus dentaires se produit. Par la suite, cet uranium se désintègre naturellement pour donner naissance, entre autres, à du thorium $230\left({ }^{230} \mathrm{Th}\right)$ dont la période de décroissance de 75200 ans peut être utilisée à des fins de datation (voir Ivanovich et Harmon, 1992). L'accumulation au cours du temps d'électrons piégés sous l'effet de la radioactivité naturelle dans des défauts du système 
cristallin des minéraux, tel que l'hydroxyapatite de l'émail dentaire, peut également être utilisée pour dater des évènements géologiques ou archéologiques via un panel de méthodes dites paléodosimétriques, dont la méthode de la résonance de spin électronique (ESR). Le nombre total d'électrons piégés dans le minéral considéré est en effet proportionnel à la dose totale de radiations qu'il a reçue au cours du temps, appelée paléodose (en grays, Gy), et à la dose naturelle de radiations reçue au cours d'une année, ou dose annuelle (en $\mu \mathrm{Gy} / \mathrm{an}$ ). Dans le cas des restes paléontologiques, la dose annuelle varie au cours du temps en fonction de l'évolution de la teneur en radioéléments de l'échantillon qui doit alors être modélisée pour permettre une estimation d'âge (Grün, 1989).

Le calcul d'un âge ESR d'un échantillon se décompose en deux parties distinctes : d'une part la détermination de la paléodose, d'autre part celle de la dose annuelle. La paléodose est déterminée par vieillissement artificiel de l'échantillon par irradiation de portions identiques de celui-ci (aliquotes) à des doses croissantes. À chaque dose d'irradiation appliquée correspond une valeur particulière de l'intensité du signal ESR. L'extrapolation de la courbe théorique de croissance de l'intensité ESR en fonction de la dose ajoutée ainsi obtenue à une intensité nulle donne la valeur de la paléodose de l'échantillon considéré (Fig. 4). La dose annuelle est quant à elle calculée à partir des teneurs en radionucléides de l'échantillon et des sédiments dans lequel il a été retrouvé, en tenant compte de la position géographique du gisement étudié.

Lors de la datation d'une dent archéologique, il faut donc modéliser l'évolution de la teneur en uranium des différents tissus dentaires au cours de la fossilisation. Pour cela, la prise en compte commune des données U-Th et ESR (modèle US, Grün et al., 1988) permet de calculer pour chaque tissu dentaire un paramètre d'incorporation $\mathrm{p}$ représentatif des données analytiques obtenues et d'en déduire les variations de dose annuelle liées à cette incorporation d'uranium au cours du temps.
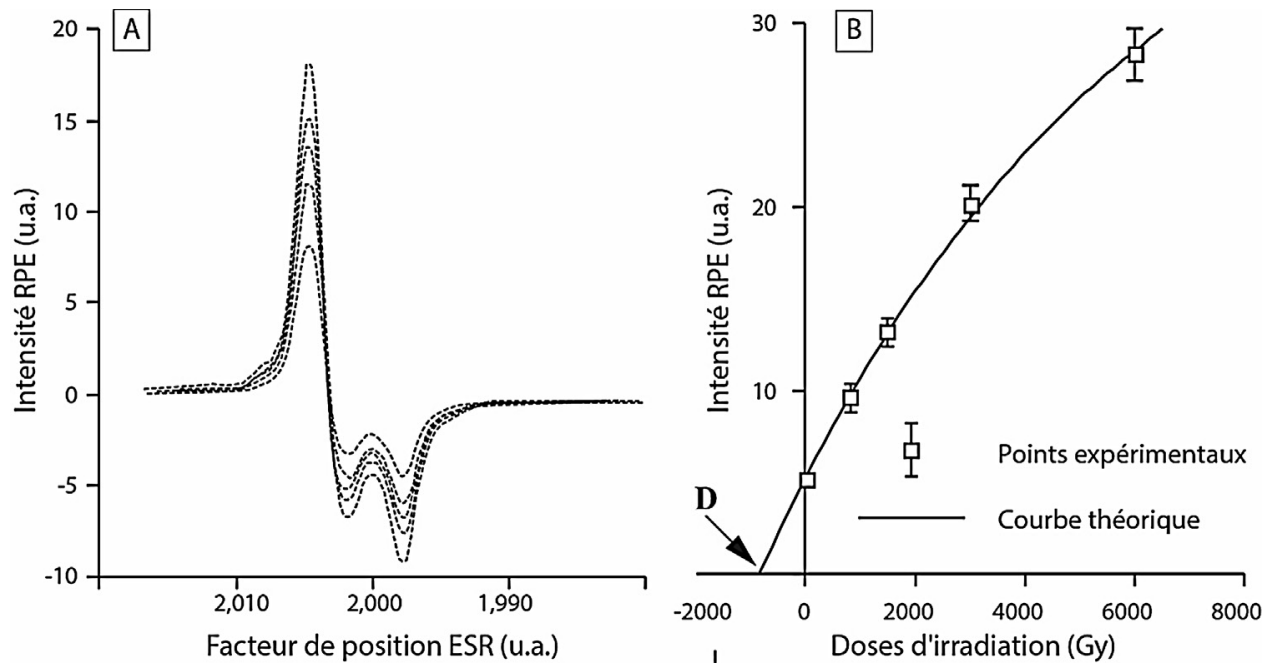

Fig. 4. Évolution de l'intensité du signal ESR d'un émail dentaire en fonction de doses d'irradiation croissantes (A) et courbe de croissance correspondante pour extrapolation de la paléodose (B).

Evolution of the intensity of tooth enamel ESR signal with increasing irradiation doses (A) and corresponding growth curve for the paleodose extrapolation $(B)$. 


\section{Présentation des gisements paléolithiques : cadre stratigraphique et nouvelles données chronologiques}

Les différentes séquences lœssiques auxquelles sont associées les occupations paléolithiques présentées dans le cadre de cette étude sont schématisées à la Fig. 2. Elles sont regroupées par région :

- Moldavie ;

- Dobrogea ;

- plaine orientale du Danube.

Précisons que l'étude du matériel lithique et l'interprétation chronologique de la plupart de ces gisements ont déjà fait l'objet de publications auxquelles nous nous réfèrerons. Par contre pour les gisements de Ripiceni Izvor et la grotte la Adam, nous disposons de nouvelles données chronologiques et biostratigraphiques inédites, qui seront présentées et détaillées dans cet article de synthèse.

Les résultats de la datation IRSL des lœss associés à ces différents gisements paléolithiques sont présentés dans le Tableau 1. Ils y sont comparés aux dates IRSL de la séquence lœssique de référence de Tuzla.

\subsection{Moldavie}

\subsubsection{Mitoc Valea Izvorului (Tuffreau et al., 2009)}

Le gisement paléolithique de Mitoc Valea Izvorului est situé sur la rive droite de la vallée du Prut, un affluent du Danube (Fig. 1), à $300 \mathrm{~m}$ du gisement aurignacien et gravettien de Mitoc Malu Galben (Otte et Chirica, 1993 ; Chirica, 2001; Otte et al., 2007). La séquence lœssique de Mitoc Valea Izvorului repose sur les graviers fluviatiles d'une ancienne terrasse du Prut, la

Tableau 1

Résultats de la datation IRSL et données sur la radioactivité des lœss associés aux gisements paléolithiques du nord-est et du sud-est de la Roumanie ainsi qu'à la séquence lœssique de référence de Tuzla (Dobrogea).

IRSL dating results and radioactivity data for the loess associated with the Palaeolithic sites from southeastern and northeastern Romania and the loessic reference sequence of Tuzla (Dobrogea).

\begin{tabular}{|c|c|c|c|c|c|c|}
\hline Site & Échantillon & $\begin{array}{l}\text { De } \pm \sigma \\
(G y)\end{array}$ & $\begin{array}{l}\text { Da } \\
(\mathrm{Gy} / \mathrm{ka})\end{array}$ & $\begin{array}{l}\text { Âge IRSL mesuré } \\
\pm \sigma(\mathrm{ka})\end{array}$ & $\begin{array}{l}\text { Âge IRSL corrigé } \\
\pm \sigma(\mathrm{ka})\end{array}$ & SIM \\
\hline Mitoc Valea Izvorului & MITOF 6 & $334 \pm 23$ & $2,55 \pm 0,21$ & $131 \pm 14$ & $160 \pm 17$ & SIM 6 \\
\hline \multirow[t]{3}{*}{ La Adam } & ADAM 1 & $162 \pm 8$ & $3,09 \pm 0,26$ & $52 \pm 5$ & $55 \pm 5$ & SIM 4 \\
\hline & ADAM 2 & $415 \pm 17$ & $3,33 \pm 0,28$ & $125 \pm 11$ & $151 \pm 13$ & SIM 6 \\
\hline & ADAM 3 & $399 \pm 24$ & $2,66 \pm 0,23$ & $150 \pm 15$ & $190 \pm 19$ & SIM 6 \\
\hline Malu Rosu & MALU 1 & $78 \pm 5$ & $3,39 \pm 0,27$ & $23 \pm 2$ & $24 \pm 2$ & SIM 2 \\
\hline Ciuperceni & VIC 1 sup & $87 \pm 7$ & $3,01 \pm 0,24$ & $29 \pm 3$ & $30 \pm 3$ & SIM 3 \\
\hline \multicolumn{7}{|l|}{ La Vii } \\
\hline & VI 08-1 & $525 \pm 71$ & $2,64 \pm 0,21$ & $199 \pm 31$ & $329 \pm 51$ & SIM 9 \\
\hline \multirow[t]{3}{*}{ Tuzla } & TUZ-1 & $161 \pm 12$ & $3,33 \pm 0,27$ & $48 \pm 5$ & $\mathbf{5 1} \pm \mathbf{5}$ & SIM 4 \\
\hline & TUZ-2 & $443 \pm 50$ & $3,32 \pm 0,27$ & $133 \pm 19$ & $163 \pm 23$ & SIM 6 \\
\hline & TUZ-3 & $567 \pm 87$ & $3,20 \pm 0,27$ & $177 \pm 31$ & $250 \pm 43$ & SIM 8 \\
\hline
\end{tabular}

De : paléodoses estimées par la méthode des doses additives (délai d'un an entre l'irradiation gamma et la lecture IRSL) ; Da : dose annuelle.

a Âges IRSL corrigés pour le fading à long terme selon le protocole de V. Mejdahl (1988, 1989). 
terrasse III culminant à $110 \mathrm{~m}$ d'altitude, soit $20 \mathrm{~m}$ au-dessus de la terrasse II de Mitoc Malu Galben (Haesaerts, 2007). Cette séquence atteint $6 \mathrm{~m}$ d'épaisseur et montre une superposition de deux dépôts lœssiques L1 et L2 (Fig. 2) séparés par un paléosol brunâtre (horizon argileux Bt) surmonté d'un horizon humifère et de dépôts limono-sableux. Le lœss inférieur L2 renferme une industrie du Paléolithique moyen (Tuffreau et al., 2009). L'âge IRSL du lœss L2 (149 ka) suggère une mise en place au SIM 6. Ce résultat démontrait ainsi pour la première fois l'existence d'occupation humaine du Paléolithique moyen attribuable au Pléistocène moyen (Tuffreau et al., 2009) remettant dès lors en question la chronologie courte du Paléolithique moyen de Roumanie (Fig. 3).

\subsubsection{Ripiceni Izvor}

3.1.2.1. Présentation du gisement (d'après Păunescu, 1993). Le gisement de Ripiceni-Izvor, aujourd'hui disparu sous les eaux à la suite de la construction d'un barrage, est situé sur la rive droite de la vallée du Prut à environ $10 \mathrm{~km}$ au sud de Mitoc Valea Izvorului. Des fouilles y ont été menées de 1961 à 1981. Il constitue un gisement de référence pour le Paléolithique de l'Est de la Roumanie en contexte lœssique. La fouille du gisement de Ripiceni-Izvor a permis d'étudier six niveaux moustériens (I à VI notés Mo I à Mo VI à la Fig. 2) surmontés de niveaux aurignaciens $(\mathrm{Au})$ et gravettiens $(\mathrm{Gr})$. Ces niveaux archéologiques sont préservés au sein d'une séquence lœssique de $10 \mathrm{~m}$ d'épaisseur reposant sur des graviers fluviatiles d'une ancienne terrasse du Prut. La séquence lœssique de Ripiceni Izvor montre une superposition de deux lœss séparés par un paléosol (horizon argileux brunâtre, horizon Bt, désigné S1 à la Fig. 2). Le lœss supérieur (noté L1 à la Fig. 2) est interstratifié de plusieurs petits paléosols beaucoup moins développés.

Les niveaux moustériens (Mo I, II) sont inclus dans le lœss inférieur, le niveau Mo III dans le paléosol S1 et les niveaux Mo IV à VI dans le lœss supérieur L1.

La méthode ${ }^{14} \mathrm{C}$ conventionnelle fut appliquée à des charbons de bois provenant des niveaux moustériens II et IV et du niveau aurignacien (Păunescu, 1993). Les dates ${ }^{14} \mathrm{C}$ suivantes, non calibrées, furent obtenues : moustérien III (46, $4+4,7 /-2,9$ ka BP ; 45 + 1,4/-1,2 ka BP ; 46,2 $\pm 1,1 \mathrm{ka})$, moustérien IV (44,8 +1,3/-1,1 ka BP ; 43,8 +1,1/-1 ka BP ; 42,5 + 1,3/ $-1,1 \mathrm{ka} \mathrm{BP} ; 40,2+1,1 /-1 \mathrm{ka} \mathrm{BP})$ et aurignacien (28,4 ka BP). Soulignons, que les âges ${ }^{14} \mathrm{C}$ des niveaux moustériens, bien que situés à la limite de la méthode, ont néanmoins été utilisés par Păunescu comme argument chronologique alors qu'ils ne constituent que des âges minimum.

En s'appuyant sur ces dates ${ }^{14} \mathrm{C}$ et sur le schéma pédostratigraphique (ou la chronologie courte) d'A. Conea (1969), Păunescu attribua l'ensemble des niveaux moustériens au Dernier Glaciaire. Le paléosol brunâtre $\mathrm{S} 1$, soit le premier sol le plus développé à partir de la surface, daté de 46-45 ka par ${ }^{14} \mathrm{C}$ et associé au niveau Mo III, était attribué au SIM 3 (interstade Moershoofd). Le niveau moustérien sus-jacent, Mo IV, était corrélé à l'interstade Hengelo (SIM 3) tandis que les niveaux moustériens inférieurs, Mo I et II étaient attribués au Début Glaciaire (SIM 5.1-5.4 ; interstades Amersfoort, Brörup et Odderade).

Le matériel de la couche 4 (ou niveau Mo IV) a été récemment redaté par ${ }^{14} \mathrm{C}$ par Doboş et Trinkaus (2012) qui ont proposé un âge minimum $>45,5 \mathrm{ka}$ BP.

Le matériel lithique et faunique (grands mammifères) de Ripiceni Izvor, conservés à l'Institut d'Archéologie « Vasile Pârvan » de Bucarest, a été réexaminé respectivement par A. Tuffreau et P. Auguste. Des dents issues des niveaux moustériens I et III ont été datées par la méthode ESR/ U-Th par J.J. Bahain et X. Shao. Les résultats de ces différentes études, inédites, sont présentés ci-dessous. 
3.1.2.2. Les industries lithiques du Paléolithique moyen (A. Tuffreau). La fouille du gisement de Ripiceni Izvor a notamment permis de reconnaître six niveaux attribués au Moustérien. A. Păunescu a défini trois ensembles distincts séparés par des couches stériles (Păunescu, 1993, 1999a). Il interpréta les trois niveaux inférieurs (I, II, III) comme appartenant à un « Moustérien typique de débitage Levallois, riche en racloirs » et datant d'environ 60-65 ka et 46-47 ka BP. Les niveaux intermédiaires (IV et V) appartiendraient au " faciès moustérien de tradition acheuléenne de débitage Levallois, riche en racloirs et avec une assez importante quantité de formes bifaciales », remontant à environ 44,8-40 ka BP pour le niveau IV et entre 40 et $37 \mathrm{ka} \mathrm{BP}$ pour le niveau V. Le dernier niveau (VI) se rattacherait à un «Moustérien denticulé de débitage Levallois, à technique bifaciale présente », datant de 36 à 35 ka BP.

Le réexamen du matériel lithique des six niveaux moustériens de Ripiceni Izvor conservé à l'Institut d'Archéologie «Vasile Pârvan » (Bucarest) montre une différence nette entre un ensemble inférieur et un ensemble supérieur, sans aucun lien phylogénétique. L'ensemble inférieur, constitué des niveaux I à III, se caractérise par la présence de nombreux nucléus Levallois (essentiellement récurrent centripète et préférentiel), et par un outillage dominé par des racloirs droits et convexes, parfois amincis. L'ensemble supérieur, constitué des niveaux IV et V, se caractérise par la présence moins prononcée des nucléus Levallois mais surtout par le grand nombre et par la grande variabilité typo-morpho-fonctionnelle des pièces bifaciales. Le niveau VI, qui comprend peu de pièces, pourrait se rattacher à l'ensemble supérieur notamment d'après la présence de Faustkeilblätter. Cet ensemble supérieur est à rapprocher d'autres industries lithiques d'Europe centrale regroupées dans les Keilmessergruppen (KMG) ou Micoquien d'Europe centrale (Bosinski, 1967, 2000-2001 ; Jöris, 2006) que l'on retrouve aussi à l'est de l'Europe (Chabai et al., 2004). Le matériel lithique des niveaux I à III de Ripiceni Izvor s'inscrit dans l'étendue chronologique des industries du Paléolithique moyen (300-40 ka) sans que l'on puisse lui attribuer une signification chronologique plus précise. Par contre, les industries lithiques (KMG) des niveaux supérieurs sont caractéristiques de la phase récente du Paléolithique moyen (130-40 ka) bien que des pièces bifaciales micoquiennes soient parfois présentes au Pléistocène moyen récent (Balescu et al., 2015 ; Haesaerts et Koulakovka, 2006).

\subsubsection{Le matériel faunique (les grands mammifères) (P. Auguste). Les vestiges de grands} mammifères provenant des différents niveaux paléolithiques de Ripiceni Izvor ont permis de mettre en évidence des assemblages fauniques qui se caractérisent par une évolution de certaines espèces permettant une approche biochronologique (Auguste, 2012). Le niveau Moustérien I comprend ainsi un mammouth laineux aux caractères dentaires principaux (épaisseur d'émail et fréquence laminaire) proches des animaux du début du Pléistocène supérieur (Tableau 2). Le mammouth du niveau Moustérien II apparaît un peu plus évolué avec une fréquence laminaire plus élevée et une épaisseur d'émail plus faible, mais avec des valeurs restant encore éloignées des formes typiques du milieu du Weichsélien. Les mammouths des autres niveaux (Moustérien III à V et Gravettien) sont eux similaires au mammouth laineux évolué weichsélien.

Associé à ce mammouth laineux dans le niveau Moustérien I, un loup de très forte taille est présent. Il se rapproche morphométriquement des loups de la fin du Pléistocène moyen et du début du Pléistocène supérieur. C'est le cas en particulier pour l'Aven de Marquet ou encore pour Villereversure, avec une denture de même proportion : pour le loup du niveau Mo I, la longueur de la première molaire inférieure est de $29 \mathrm{~mm}$, la largeur de 11,5 mm ; pour Arquet, la longueur est de 28,17, la largeur de 11,44 (Boudadi-Maligne, 2010). 
Tableau 2

Comparaisons des mesures des molaires supérieures des mammouths laineux des niveaux Moustérien Mo I et Mo II de Ripiceni Izvor avec celles de mammouths du Pléistocène moyen et du Pléistocène supérieur.

Comparison between the upper molar measurements on the woolly mammoths from the Mousterian levels Mo I and Mo II of Ripiceni Izvor and those on the Middle and Upper Pleistocene mammoths.

\begin{tabular}{lll}
\hline Mammuthus primigenius, M2 sup & E & F \\
\hline Ripiceni Izvor Mo I & 1,5 & 8 \\
Ripiceni Izvor Mo II & 1,3 & 8,5 \\
M. trogontheri Hanhoffen (1) & $2,14(1,9-2,3)$ & $6,79(5,5-8)$ \\
M. primigenius Zemst IIB (2) & $1,7(1,5-1,9)$ & $8,8(6-11)$ \\
M. primigenus Hanhoffen (1) & $1,55(1-2,2)$ & $10(8-13)$ \\
\hline
\end{tabular}

E : épaisseur d'émail ; F : fréquence laminaire (nombre de lames $/ 10 \mathrm{~cm}$ ). Valeur moyenne suivie des minima et maxima. (1) Louguet-Lefèvre, 2005 ; (2) Germonpré, 1993.

Un cheval de forte stature est également présent dans les niveaux Mo I et Mo II. Il s'apparente assez bien avec les premiers chevaux du Pléistocène supérieur. Ainsi, par exemple, les dimensions de l'articulation proximale du métacarpien III du cheval des niveaux Mo I et II $(60 \times 39,8 \mathrm{~mm})$ sont comparables à celles des chevaux de Zemst II B $(57,1 \times 38,4 \mathrm{~mm})$ (Germonpré, 1993). Dès le niveau Moustérien III, un cheval plus gracile est identifié, il se rapproche des formes connues durant le Weichsélien, et en particulier d'Equus germanicus. Cette espèce est identifiée également en Bulgarie et témoigne donc de parallèles paléobiogéographiques cohérents dans une certaine mesure avec l'Europe occidentale (Guadelli et Delpech, 2000).

L'association faunique des niveaux Moustérien I et II de Ripiceni Izvor semble donc témoigner d'une formation de ces niveaux durant une phase ancienne du Pléistocène supérieur. Comparée à ses homologues nord-ouest européens (Auguste, 2009) et d'Europe orientale (Markova, 2007), elle s'inscrit bien dans le complexe mammalien Shkurlatien correspondant au SIM 5 (mammouth laineux archaïque, cheval de forte taille, loup de forte taille).

3.1.2.4. Les résultats de la datation ESR/U-Th (J.J. Bahain et Q. Shao). Afin de tenter de positionner chronologiquement le site de Ripiceni Izvor et bien que ce gisement ne soit plus accessible et qu'une réelle étude dosimétrique n'ait pu y être menée, plusieurs dents de cheval provenant de deux niveaux archéologiques (Moustérien I et Moustérien III) ont été analysées par les méthodes de la résonance de spin électronique et du déséquilibre dans les familles de l'uranium combinées (ESR/U-Th) selon le protocole expérimental décrit dans Bahain et al. (2002).

Au laboratoire, après extraction de lamelles d'émail, ces dernières ont été nettoyées au moyen d'une fraise de dentiste afin d'éliminer toute contamination par du sédiment, du cément ou de la dentine. L'épaisseur de l'émail doit être précisément mesurée avant, au cours et après la préparation, pour que la portion d'émail éliminée lors de celle-ci puisse être prise en compte dans la détermination de la dose annuelle.

Les activités des différents radionucléides présents dans les tissus dentaires (essentiellement ${ }^{238} \mathrm{U},{ }^{226} \mathrm{Ra}$ et ${ }^{222} \mathrm{Rn}$ ) ont été mesurées par spectrométrie gamma, puis les tissus analysés par UTh (MC-ICP-MS) selon le protocole détaillé dans Shao et al. (2011), ce qui a permis d'obtenir les rapports ${ }^{234} \mathrm{U} /{ }^{238} \mathrm{U}$ et ${ }^{230} \mathrm{Th} /{ }^{234} \mathrm{U}$, indispensables au calcul des facteurs d'incorporation pour chaque tissu (Tableau 3). Cette double analyse permet également d'estimer, en croisant les 
Tableau 3

Données U-Th obtenues par MC-ICP-MS, rapports ${ }^{222} \mathrm{Rn} /{ }^{230}$ Th déterminés par croisement des données de MC-ICP-MS et gamma et épaisseurs (avant et après préparation) utilisées pour le calcul d'âge par la méthode combinée ESR/U-Th pour les dents analysées provenant du site de Ripiceni Izvor.

U-Th data obtained by MC-ICP-MS, ${ }^{222} R n{ }^{230 T h}$ ratio determined by the MC-ICP-MSand gamma-ray cross data and thickness (before and after preparation) used for the age calculation by the ESR/U-series method for the analyzed teeth collected at Ripiceni Izvor

\begin{tabular}{|c|c|c|c|c|c|c|c|c|c|}
\hline Échantillon & Tissu & $\begin{array}{l}\mathrm{U} \\
(\mathrm{ppm})\end{array}$ & ${ }^{230} \mathrm{Th} /{ }^{232} \mathrm{Th}$ & ${ }^{234} \mathrm{U} /{ }^{238} \mathrm{U}$ & ${ }^{230} \mathrm{Th} /{ }^{234} \mathrm{U}$ & ${ }^{222} \mathrm{Rn} /{ }^{230} \mathrm{Th}$ & $\begin{array}{l}\text { Épaisseur } \\
\text { initiale } \\
(\mu \mathrm{m})\end{array}$ & $\begin{array}{l}\text { Épaisseur } \\
\text { enlevée côté } \\
\text { interne }(\mu \mathrm{m})\end{array}$ & $\begin{array}{l}\text { Épaisseur } \\
\text { enlevée côté } \\
\text { externe }(\mu \mathrm{m})\end{array}$ \\
\hline RI1202 & Émail & $7,684 \pm 0,026$ & $1,240 \pm 0,003$ & $>100$ & $0,160 \pm 0,001$ & 0,511 & $1337 \pm 167$ & $145 \pm 18$ & $133 \pm 17$ \\
\hline (RP837 $n^{\circ} 14$ Moust III) & Dentine & $102,200 \pm 0,906$ & $1,198 \pm 0,005$ & $>100$ & $0,112 \pm 0,001$ & 0,279 & & & \\
\hline RI1203 & Émail & $1,653 \pm 0,005$ & $1,251 \pm 0,003$ & $>100$ & $0,216 \pm 0,001$ & 1,000 & $1336 \pm 167$ & $150 \pm 19$ & $63 \pm 8$ \\
\hline \multirow{2}{*}{ (RP837 n 14 Moust III) } & Dentine & $65,592 \pm 1,054$ & $1,211 \pm 0,007$ & $>100$ & $0,060 \pm 0,001$ & 1,000 & & & \\
\hline & Cément & $135,745 \pm 2,433$ & $1,245 \pm 0,049$ & $>100$ & $0,455 \pm 0,009$ & 0,183 & & & \\
\hline RI1204 & Émail & $1,657 \pm 0,005$ & $1,293 \pm 0,003$ & $>100$ & $0,272 \pm 0,001$ & 1,000 & $989 \pm 124$ & $166 \pm 21$ & $108 \pm 14$ \\
\hline (RP1968 514 Moust I) & Dentine & $338,399 \pm 11,187$ & $1,237 \pm 0,017$ & $>100$ & $0,240 \pm 0,009$ & 0,164 & & & \\
\hline RI1205 & Émail & $2,557 \pm 0,012$ & $1,279 \pm 0,004$ & $>100$ & $0,206 \pm 0,001$ & 1,000 & $1349 \pm 169$ & $130 \pm 16$ & $136 \pm 17$ \\
\hline $\begin{array}{l}\left(\mathrm{G} 23 \mathrm{n}^{\circ} 89 \text { inv }\right. \\
1470 / 5034 \text { Moust I) }\end{array}$ & Dentine & $166,093 \pm 7,457$ & $1,240 \pm 0,011$ & $>100$ & $0,124 \pm 0,003$ & 0,426 & & & \\
\hline
\end{tabular}



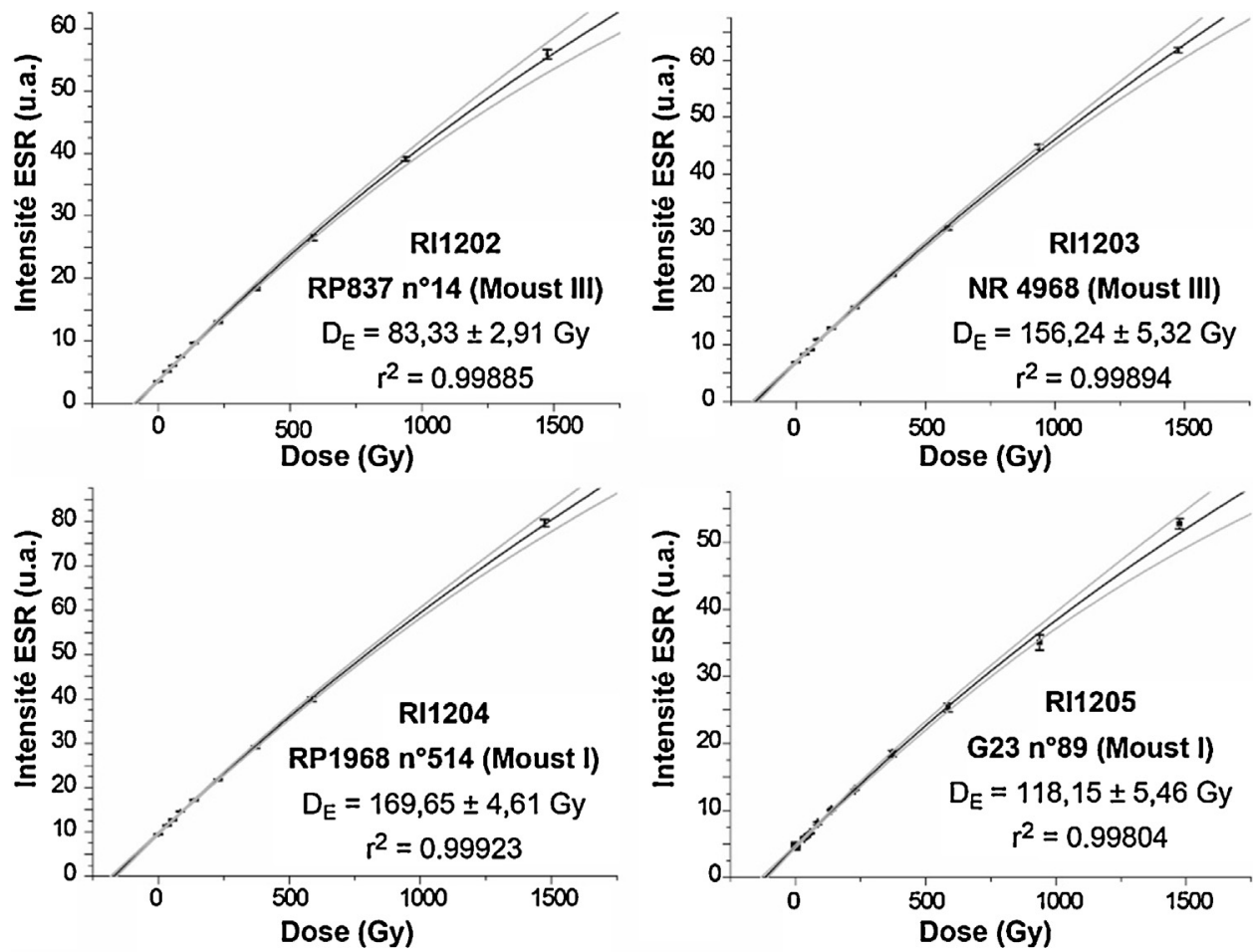

Fig. 5. Courbes de croissance des dents de Ripiceni Izvor. Growth curves for the Ripiceni Izvor teeth.

données MC-ICP-MS et $\gamma$, les éventuelles fuites de radon-222, élément très mobile dont les descendants sont d'importants émetteurs $\alpha, \beta$ et $\gamma$ (Bahain et al., 1992) (Tableau 3).

L'émail a ensuite été broyé mécaniquement, puis tamisé. La fraction 100-200 $\mu \mathrm{m}$ récupérée lors de ce tamisage a été séparée en dix aliquotes. Neuf de celles-ci ont été irradiées au CEA (CEN Saclay) à l'aide d'une source gamma au ${ }^{60} \mathrm{Co}$ à des doses de $34,55,85,137,228,371,586$, 937 et 1475 Gy. Les intensités ESR des dix aliquotes ont ensuite été mesurées avec un spectromètre Bruker EMX, puis les courbes de croissance intensités/doses ont été tracées et les paléodoses déterminées (Fig. 5).

Les activités en radionucléides du sédiment utilisées pour estimer l'âge des dents de Ripiceni Izvor sont les suivantes : $\mathrm{U}=2,17 \pm 0,29 \mathrm{ppm} ; \mathrm{Th}=8,12 \pm 1,10 \mathrm{ppm} ; \mathrm{K}=1,43 \pm 0,16 \%$ (Sanda Balescu, comm. pers.). Ces teneurs ont été mesurées par activation neutronique. Il s'agit d'une valeur moyenne calculée à partir de mesures effectuées sur 8 échantillons de lœss prélevés à proximité de Ripiceni Izvor au sein des séquences lœssiques de Mitoc Malu Galben et de Mitoc Izvorului. Ces valeurs sont comparables à celles obtenues pour l'ensemble des lœss (L1, L2 et L3) au sein des trois séquences lœssiques de référence (Tuzla, Mircea Voda et Mostistea) : $\mathrm{U}=3,1 \pm 0,3 \mathrm{ppm} ; \mathrm{Th}=9,8 \pm 0,9 \mathrm{ppm} ; \mathrm{K}=1,56 \pm 0,2 \%$. Pour la teneur en eau de ce sédiment, une valeur de $20 \pm 5 \%$ équivalente à celle estimée pour les lœss de Mitoc Malu Galben et Mitoc Izvorului (Balescu et al., 2003) a été retenue. Enfin, pour les âges ESR/U-Th, les différentes contributions à la dose annuelle et les paramètres d'incorporation de l'uranium pour chaque tissu dentaire ont été calculés, en utilisant les facteurs de conversion teneurs-doses d'Adamiec et Aitken (1998). Les âges ESR/U-Th, les différentes contributions à la dose annuelle 
Tableau 4

Paléodoses, paramètres d'incorporation, contributions des différents rayonnements au débit de dose annuel et âges ESR/U Th obtenus pour les dents analysées de Ripiceni Izvor.

Paleodoses, U-uptake parameters, contributions of the different rays to the dose rate and ESR/U series ages obtained for the analyzed teeth of Ripiceni Izvor.

\begin{tabular}{|c|c|c|c|c|c|c|c|c|}
\hline Échantillon & Tissu & $\begin{array}{l}\text { Paléodose } \\
\text { (Gy) }\end{array}$ & $\begin{array}{l}\text { Paramètre } \\
\text { d'incorporation } \\
\mathrm{p} \text { (US) }\end{array}$ & $\begin{array}{l}D_{\alpha} \text { interne moyenne }{ }^{a} \\
(\mu \text { Gy/an })\end{array}$ & $\begin{array}{l}D_{\beta} \text { externe moyenne }{ }^{b} \\
(\mu \text { Gy/an })\end{array}$ & $\begin{array}{l}D(\gamma+\operatorname{cosm}) \\
\text { externe }^{c} \\
(\mu \text { Gy/an })\end{array}$ & $\begin{array}{l}\mathrm{D}_{\mathrm{a}} \\
\text { moyenne } \\
(\mu \mathrm{Gy} / \mathrm{an})\end{array}$ & $\begin{array}{l}\text { Age } \\
\text { RPE/U-Th } \\
\text { (AU) (ka) }\end{array}$ \\
\hline $\begin{array}{l}\text { RI1202 } \\
\text { (RP837 n }{ }^{\circ} 14 \text { Moust III) }\end{array}$ & $\begin{array}{l}\text { Émail } \\
\text { Dentine }\end{array}$ & $83,33 \pm 2,91$ & $\begin{array}{l}1,5478 \pm 0,2998 \\
3,2524 \pm 0,4365\end{array}$ & $133 \pm 73$ & $199 \pm 54$ & $842 \pm 57$ & $1174 \pm 107$ & $71 \pm 6$ \\
\hline $\begin{array}{l}\text { RI1203 } \\
(\text { RP837 n } 14 \text { Moust III) }\end{array}$ & $\begin{array}{l}\text { Émail } \\
\text { Dentine } \\
\text { Cément }\end{array}$ & $156,24 \pm 5,32$ & $\begin{array}{l}1,5745 \pm 0,3690 \\
12,6915 \pm 1,5788 \\
-0,5331 \pm 0,1289\end{array}$ & $91 \pm 25$ & $602 \pm 161$ & $839 \pm 57$ & $1532 \pm 173$ & $102 \pm 11$ \\
\hline $\begin{array}{l}\text { RI1204 } \\
\text { (RP1968 } 514 \text { Moust I) }\end{array}$ & $\begin{array}{l}\text { Émail } \\
\text { Dentine }\end{array}$ & $169,65 \pm 4,61$ & $\begin{array}{l}0,3777 \pm 0,2993 \\
0,7480 \pm 0,3524\end{array}$ & $154 \pm 50$ & $943 \pm 257$ & $831 \pm 57$ & $1928 \pm 268$ & $88 \pm 12$ \\
\hline $\begin{array}{l}\text { RI1205 } \\
\text { (G23 } \mathrm{n}^{\circ} 89 \\
\text { inv } 1470 / 5034 \text { Moust I) }\end{array}$ & $\begin{array}{l}\text { Émail } \\
\text { Dentine }\end{array}$ & $118,15 \pm 5,46$ & $\begin{array}{l}1,6355 \pm 0,3203 \\
4,4517 \pm 0,5836\end{array}$ & $144 \pm 80$ & $236 \pm 76$ & $826 \pm 57$ & $1206 \pm 124$ & $98 \pm 9$ \\
\hline
\end{tabular}

\footnotetext{
${ }^{a}$ Un facteur $\mathrm{k}$ (efficacité alpha) de 0,13 $\pm 0,02$ a été utilisé (Grün et Katzenberger-Apel, 1994).

${ }^{\mathrm{b}}$ Les valeurs d'atténuation beta dans l'émail ont été calculées en utilisant une approche Monte-Carlo (Brennan et al., 1997).

${ }^{c}$ La dose cosmique a été calculée à partir de la profondeur de chaque échantillon en utilisant les tables de Prescott et Hutton (1994).

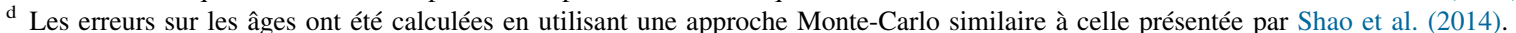


et les facteurs d'incorporation obtenus pour les échantillons de Ripiceni Izvor sont présentés dans le Tableau 4.

Même si on ne peut pas qualifier les résultats obtenus de « datations » en raison de la faiblesse de la reconstruction paléodosimétrique (des teneurs moyennes en radionucléides ont été utilisées pour estimer l'âge de dents provenant de deux niveaux distincts, aucune mesure de dosimétrie gamma in situ n'a pu être réalisée et la teneur en eau originelle du sédiment n'a pas pu être estimée), on peut noter que les paléodoses mesurées pour les dents de Ripiceni Izvor, comprises entre 80 et $170 \mathrm{~Gy}$, sont relativement faibles, alors que les teneurs en uranium des tissus dentaires analysés, de 1,6 à 7,7 ppm dans les emails et de 65 à 338 ppm dans les dentines et les céments, sont relativement importantes. En conséquence, ces données ne permettent pas d'obtenir des estimations d'âge plus anciennes que $115 \mathrm{ka}$. Ces âges suggèrent donc une mise en place des niveaux moustériens correspondants (Mo I et III) lors de la seconde moitié du SIM 5, c'est-à-dire lors du Début Glaciaire (SIM 5.4-5.1).

3.1.2.5. Nouvelle interprétation chronologique des niveaux moustériens. Les résultats de l'analyse morphométrique des grands mammifères associés aux niveaux moustériens inférieurs, Mo I à III, excluent une attribution au SIM 6. Ils suggèrent plutôt un âge Début Glaciaire en accord avec les âges ESR/U-Th des dents associées à ces mêmes niveaux moustériens. Ces nouveaux arguments biostratigraphiques et chronologiques confortent dès lors l'attribution des niveaux moustériens inférieurs I et II au Début Glaciaire (SIM 5.4-5.1) tel que l'avait suggéré Păunescu (1993), mais remettent par contre en question l'âge SIM 3 (interstade de Moershoofd) du niveau Mo III.

Le réexamen de l'industrie lithique des six niveaux moustériens montre une différence nette entre l'ensemble inférieur (comprenant les niveaux Mo I à III) et l'ensemble supérieur (Mo IV et V), mais ne fournit aucun argument chronologique, l'industrie lithique des niveaux inférieurs étant présente aussi bien à la fin du Pléistocène moyen récent (SIM 6) qu'au début du Pléistocène supérieur.

Enfin, ces nouvelles données chronologique et biostratigraphique, permettent de proposer une corrélation du sol de Ripiceni au pédocomplexe S1 qui, au sein des séquences lœssiques de référence du SE de la Roumanie (Tuzla, Mostiştea et Mircea Vodă), est attribué à l'ensemble du SIM 5 (SIM 5.5 à 5.1). Ce pédocomplexe S1 regroupe plusieurs horizons pédologiques superposés parfois séparés par un mince dépôt lœssique ou limoneux (Conea, 1969). Ces épisodes successifs de pédogénèse ont été également révélés par l'enregistrement de la susceptibilité magnétique (Buggle et al., 2009 ; Fitzsimmons et al., 2012). L'attribution du pédocomplexe S1 à l'ensemble du SIM 5 (SIM 5,5 à 5,1) est par ailleurs démontrée par les dates OSL sur quartz obtenues à Mostiştea (Vasiliniuc et al., 2012) et à Costineşti près de Tuzla (Constantin et al., 2014) qui sont comprises entre 63 et $120 \mathrm{ka}$.

\subsection{Dobrogea}

\subsubsection{Mamaia Sat (Balescu, 2013 ; Balescu et al., 2015)}

Le gisement de Mamaia Sat (Fig. 1), étudié dans les années 1960-1980 par Păunescu (1999b) et Valoch (1968) est situé en Dobrogea, à proximité de Tuzla et Constanţa, le long du canal reliant la Mer Noire au Danube, à $500 \mathrm{~m}$ du littoral. Ce site est aujourd'hui inaccessible et n'a donc pas pu faire l'objet de nouvelles fouilles. La coupe est caractérisée par une séquence de $19 \mathrm{~m}$ de lœss (Fig. 2). Elle montre une succession de cinq lœss et de quatre paléosols intercalés (A, B, C, D renommés ici respectivement $\mathrm{S} 1, \mathrm{~S} 2.1, \mathrm{~S} 2.2, \mathrm{~S} 3$ à la Fig. 2). Les paléosols $\mathrm{S} 1, \mathrm{~S} 2.1, \mathrm{~S} 2.2$ sont 
brunâtres tandis que le pédocomplexe $\mathrm{S} 3$, situé à la base de la séquence, est rougeâtre (très rubéfié).

Le site multistratifié de Mamaia Sat a livré deux niveaux d'industrie lithique attribués par Păunescu (1999b) et Valoch $(1968,1993)$ au Paléolithique moyen : les assemblages I et II respectivement dans le sol brunâtre inférieur (S2.2) et le sol rougeâtre (S3). En se référant au schéma chronostratigraphique de Conea (1969), le sol rougeâtre S3 avait été attribué par Păunescu et Valoch au Dernier Interglaciaire (SIM 5). Les paléosols brunâtres sus-jacents (S2.2, S2.1 et S1), étaient corrélés à des interstades du Dernier Glaciaire. Les deux assemblages du Paléolithique moyen I et II, étaient dès lors rapportés respectivement à la Dernière Glaciation et au Dernier Interglaciaire.

En s'appuyant sur l'enregistrement pédostratigraphique et la chronologie IRSL des séquences lœssiques de référence du SE de la Roumanie (Tuzla, Mostiştea et Mircea Vodă), une nouvelle interprétation chronologique de la séquence de Mamaia Sat (Fig. 2) a été proposée (Balescu, 2013 ; Balescu et al., 2015). Le premier sol brunâtre S1 correspondrait au SIM 5. Les deux sols brunâtres sous-jacents, S2.1 et S2.2, seraient attribués au SIM 7 ; ils seraient l'équivalent du pédocomplexe S2 qui au sein des séquences lœssiques du SE de la Roumanie est systématiquement dédoublé (S2.1 et S2.2) (bipartition du SIM 7 ; Balescu et al., 2010). Enfin, le sol rougeâtre S3 correspondrait au SIM 9.

En conséquence, les industries paléolithiques de Mamaia Sat préservées dans les paléosols S2.2 et S3 pourraient remonter aux SIM 7 et SIM 9. Nous aurions donc à Mamaia Sat les témoins d'une occupation du Paléolithique moyen antérieur au Dernier Interglaciaire (SIM 5), qui pourrait remonter au SIM 9.

Le matériel lithique a également été réexaminé par A. Tuffreau (in Balescu et al., 2015). Ces industries ne montrent aucune caractéristique diagnostique du Paléolithique moyen à l'exception des pièces foliacées et de quelques nucléus et éclats Levallois. La présence de pièces foliacées au sein de l'assemblage I (paléosol S2.2) de Mamaia Sat est cohérente avec celles trouvées en Ukraine à Korolovo (assemblage Va) dans le paléosol V supérieur dont la position stratigraphique suggère un âge SIM 7-fin SIM 8 (Haesaerts et Koulakovka, 2006). Des pièces moustériennes à débitage Levallois ont également été mises au jour à Kozarnika en Bulgarie, dans la couche 10b rapportée au SIM 6 (130-200 ka) (Guadelli et al., 2005). Le débitage Levallois datant du SIM 7 est par ailleurs déjà connu dans différents sites de Podolie en particulier à VelykyiGlybochok où a été découvert le plus ancien assemblage paléolithique moyen de Podolie (Sytnik, 2000 ; Lanczont et al., 2014). Ce dernier, à débitage Levallois, a été mis au jour dans le paléosol inférieur S2 II du pédocomplexe S2 dédoublé (S2 I et S2 II) attribué au SIM 7 et daté $235 \pm 22$ ka par la méthode post-IR IRSL.

\subsubsection{La grotte de La Adam (Tuffreau et al., 2013 ; Balescu, 2013)}

3.2.2.1. Présentation du gisement (Tuffreau et al., 2013 ; Balescu, 2013). La grotte La Adam située sur le plateau de la Dobrogea, (Fig. 1) a été fouillée par des paléontologues dans les années 1950-1960 (Radulescu et Samson, 1962 ; Samson, 1971 ; Petculescu et Samson, 2001). Aucun préhistorien n'ayant été associé à ces fouilles, les données concernant le matériel lithique demeuraient très faibles. Un niveau d'occupation paléolithique moyen a été fouillé à l'entrée de la grotte, de 2006 à 2009, par A. Tuffreau et R. Dobrescu (Tuffreau et al., 2013).

L'entrée de la grotte a été subdivisée en deux secteurs :

- AG (extérieur) ;

- EG (intérieur) (Tuffreau et al., 2013). 
Dans le secteur AG, ont été individualisées deux couches lœssiques (notées L2 et L1, Fig. 2). Il s'agit de poussières éoliennes en provenance de l'extérieur, qui ont été piégées à l'entrée de la grotte pendant les phases froides et arides des périodes glaciaires. Ces lœss L1 et L2 sont encadrés de dépôts limoneux riches en fragments ou débris de roche calcaires d'origine thermoclastique. Ils sont par ailleurs séparés par un paléosol brunâtre (horizon Bt tronqué, « couche D1 ») surmonté d'un limon humifère à débris clastiques dispersés ( « couches D et C»). Notons que dans le secteur AG, la « couche E » de Tuffreau et al. (2013) regroupe le lœss L2 et les dépôts thermoclastiques sous-jacents (que nous désignerons par « dépôt thermoclastique inférieur ») (Fig. 2).

À l'intérieur de la grotte (secteur EG) par contre, où furent réalisées les fouilles, les lœss L1 et L2 sont absents (voir Fig. 4 de Balescu, 2013). La « couche E n'est donc constituée ici que de dépôts thermoclastiques. L'horizon Bt («couche D1») est partiellement, voire totalement, érodé suite à la mise en place du limon humifère sus-jacent ( « couche $\mathrm{D}$ ») qui colmate une dépression. Cette « couche $\mathrm{D}$ » repose donc soit directement sur la « couche $\mathrm{E}$ », soit sur un résidu du paléosol fortement tronqué (« couche D1»).

Enfin, à la transition entre les secteurs AG et EG, une couche thermoclastique supplémentaire a été individualisée entre le lœss L2 et le paléosol D1 (que nous désignerons par " dépôt thermoclastique supérieur »). À l'emplacement de la fouille (secteur EG), ce « dépôt thermoclastique supérieur » repose directement sur le « dépôt thermoclastique inférieur »; les deux sont confondus, indifférenciables, l'ensemble formant la « couche E ». Nous la désignerons par « dépôt thermoclastique indifférencié ».

Dans le secteur EG, les limons humifères ( « couches $\mathrm{C}$ et $\mathrm{D}$ ») qui surmontent le paléosol D1 ont livré deux niveaux du Paléolithique moyen. Quelques éclats et un racloir ont également été mis au jour dans le « dépôt thermoclastique indifférencié » (« couche E ») antérieur au paléosol D1 (Tuffreau et al., 2013).

3.2.2.2. Datation IRSL (S. Balescu et M. Lamothe). Trois échantillons destinés à la datation IRSL ont été prélevés dans le secteur AG, au sein du lœss L1 (ADAM 1) et du lœss L2 (ADAM 2 et 3). La date IRSL obtenue pour ADAM $2(151 \pm 13 \mathrm{ka})$ a été publiée (Balescu, 2013). Les deux autres échantillons ont été datés ultérieurement (données inédites). Les âges IRSL des échantillons ADAM 2 (151 ka) et ADAM 3 (190 ka) suggèrent une mise en place du lœss L2 à l'Avant Dernier Glaciaire (SIM 6). Par contre, le lœss L1 qui surmonte les couches archéologiques (D et C), daté $55 \mathrm{ka}$, est attribuable au SIM 4 (Pléniglaciaire inférieur). Le paléosol brunâtre (horizon $\mathrm{Bt}$, « couche D1 ») représente dès lors le sol du Dernier Interglaciaire (SIM 5.5). Les couches archéologiques sus-jacentes (« couches D et C »), surmontées d'un lœss du SIM 4, sont donc attribuables au Début Glaciaire (SIM 5.4-5.1). L'ensemble des horizons pédologiques (sol brunâtre et horizons humifères sus-jacents correspondant aux couches D1, D, C) sont par conséquent corrélables au pédocomplexe S1 qui, au sein des séquences lœssiques de référence du SE de la Roumanie, couvre l'ensemble du SIM 5 (SIM 5.5 à 5.1).

3.2.2.3. Les micromammifères (Arvicola terrestris) (A. Petculescu). Dans le secteur EG, à l'emplacement de la fouille, 10 espèces différentes de micromammifères ont été identifiées au sein des couches B, C, D, E et F. Elles ont été répertoriées dans Tuffreau et al. (2013). Leur répartition au sein de chacune de ces couches a fourni de précieuses informations sur le contexte paléoclimatique. 
Parmi ces espèces, notons la présence d'un fossile stratigraphique, Arvicola terrestris, au sein des couches $\mathrm{E}$ (« dépôt thermoclastique indifférencié ») et $\mathrm{C}$ (couche archéologique, limon humifère).

La mesure de l'épaisseur de l'émail dentaire de la première molaire de la mandibule (M1) d'Arvicola permet de calculer un indice SDQ selon la méthode décrite par Kolfschoten (1990). Cet indice qui diminue avec le temps, est fréquemment utilisé en Europe comme argument biostratigrahique (Kolfschoten, 1990, 1992). Notons par ailleurs qu'Arvicola terrestris est apparu en Europe au SIM 5.

À La Adam, les valeurs moyennes des indices SDQ mesurés sur les dents d'Arvicola terrestris prélevées à l'emplacement de la fouille, dans les couches E et C, sont respectivement de 92,6 $(\mathrm{n}=2)$ et 88,7 $(\mathrm{n}=2)$. Ces mesures ont été effectuées sur LRA4 ( « lingual reentrant angles-4) ») pour chaque spécimen (d'après Röttger, 1987). Notons que ces valeurs ne sont pas significativement différentes et ne reposent chacune que sur 2 dents. En se référant aux valeurs SDQ d'Arvicola répertoriées par Kolfschoten (1992) en Europe, et aux valeurs SDQ obtenues en Roumanie en contexte karstique (Petculescu, 2003), un âge Eemien/Début Glaciaire Weichsélien est ici proposé.

La valeur SDQ moyenne obtenue pour la couche archéologique $\mathrm{C}$ suggère un âge Début Glaciaire Weichsélien (SIM 5.1-5.4) qui est stratigraphiquement cohérent avec l'âge IRSL du lœss sus-jacent L1 (SIM 4).

L'âge éemien de la microfaune prélevée dans la couche $\mathrm{E}$ (« dépôt thermoclastique indifférencié ») sous le paléosol éemien (couche D1), demeure par contre problématique. L'interprétation est plus délicate et reste à ce stade de nos recherches hypothétique car nous ne disposons pas de contrôle chronologique pour la couche E (« dépôt thermoclastique indifférencié ») qui regroupe à l'emplacement de la fouille, le dépôt thermoclastique supérieur (postérieur au lœss L2; fin SIM 6-début SIM 5 ?) et le dépôt thermoclastique inférieur (antérieur au lœss L2 ; début SIM 6 ?).

Deux hypothèses peuvent donc être avancées pour tenter d'expliquer l'âge SIM 5 de la microfaune issue de la couche E, sous-jacente au paléosol éemien D1 :

- soit les dents sont issues de la partie supérieure de la couche $\mathrm{E}$ (le « dépôt clastique supérieur ») mis en place entre le lœss L2 et le paléosol D1, et pourraient être attribuées au début de l'Eemien ;

- soit la microfaune n'est pas en place, sa présence résulte de processus de bioturbation ; des rongeurs d'âge éemien ou début glaciaire, présents dans le paléosol éemien D1 ou la couche archéologique $\mathrm{D}$ du début glaciaire, auraient pénétré dans la couche E sous-jacente, d'âge SIM 6 , en y creusant des terriers.

Ces conclusions sont cohérentes avec la découverte d'Arvicola terrestris dans les niveaux moustériens préservés au sein des dépôts de grotte de l' « Abri 122 » en Roumanie (Carpates orientales) qui remonteraient au SIM 5, voire un peu avant le SIM 5 (Cosac et al., 2017 ; Veres et al., 2017).

3.2.2.4. Interprétation chronologique de l'industrie du Paléolithique moyen. L'âge Début Glaciaire Weichsélien des niveaux d'occupation paléolithique moyen associés aux couches D et C (limons humifères) surmontant le paléosol D1 est ici démontré par :

- les âges IRSL des lœss L1 (SIM 4) et L2 (SIM 6) qui encadrent les couches archéologiques D et $\mathrm{C}$; 
- les valeurs de l'indice SDQ mesuré sur des dents d'Arvicola terrestris issues de la couche C.

L'âge des artefacts issus de la couche E reste par contre imprécis. Ils sont antérieurs au paléosol éemien mais, en l'absence du lœss L2 daté par IRSL qui constitue un excellent repère chronologique, leur attribution au SIM 6 ou au début SIM 5 reste indéterminée.

\subsection{Plaine orientale du Danube}

\subsubsection{Giurgiu Malu Roşu (Alexandrescu et al., 2004) et Ciuperceni LaVii (Tuffreau et al., 2014)}

Les gisements de Giurgiu Malu Roşu et de Ciuperceni La Vii situés en bordure du Danube, dans le SE de la Roumanie, ont livré une industrie lithique attribuée respectivement à un «Aurignacien tardif » et à une phase ancienne du Paléolithique supérieur. Dans les deux cas, le matériel correspond à un faciès d'atelier préservé au sein de lœss mis en place au cours du Dernier Glaciaire (L1) (Fig. 2).

À Giurgiu-Malu Roşu, le lœss L1 renferme deux niveaux d'occupation qui ont été attribués à un Aurignacien «tardif ». Ce lœss repose sur des graviers fluviatiles d'une basse terrasse du Danube («terrasse de Giurgiu »). Plusieurs foyers en place ont été identifiés au sein du niveau archéologique inférieur. Deux échantillons de charbon de bois, issus de ces foyers, ont été datés par ${ }^{14} \mathrm{C}$ (21-23 ka BP non calibrés). L'échantillon destiné la datation IRSL (MALU1) a été prélevé directement sous le niveau archéologique daté par ${ }^{14} \mathrm{C}$. L'âge IRSL obtenu ( $\left.27 \pm 3 \mathrm{ka}\right)$ est parfaitement cohérent avec les dates ${ }^{14} \mathrm{C}$ (Alexandrescu et al., 2004 ; Balescu et al., 2010). Cette application nous a permis de tester la fiabilité de la méthode IRSL appliquée aux lœss roumains.

À Ciuperceni La Vii, quatre niveaux archéologiques ont été observés dans le lœss L1 qui colmate une dépression entamant un paléosol rougeâtre. L'ensemble de la séquence repose sur les dépôts fluviatiles de la «basse » terrasse du Danube.

En l'absence de matière organique et de restes fauniques, la mise en œuvre de la méthode IRSL s'avérait ici indispensable pour préciser l'âge de l'industrie. Les échantillons destinés à la datation IRSL ont été prélevés dans le lœss, sous le deuxième niveau archéologique (VIC-1sup) et dans le paléosol rougeâtre (VI08-1) situé à la base de la séquence.

L'âge IRSL du lœss est de $30 \pm 3 \mathrm{ka}$. L'âge IRSL du paléosol rougeâtre est de $329 \pm 51 \mathrm{ka}$. Ce paléosol est donc vraisemblablement l'équivalent du sol rougeâtre S3 observé au sein des coupes de Tuzla, Mircea Voda et Mostiştea (Balescu et al., 2010).

\section{Discussion et conclusion}

Les principaux apports de l'étude chronologique des gisements paléolithiques de l'Est de la Roumanie sont les suivants :

- l'âge des gisements du Paléolithique supérieur de Malu Roşu et Ciuperceni dans la plaine orientale du Danube, a pu être confirmé voire précisé en l'absence de matière organique, par la datation IRSL des lœss qui leur sont associés ;

- la mise en œuvre de la datation IRSL et ESR/U-Th couplée à l'analyse de la macrofaune et de la microfaune (arguments biostratigraphiques), nous a permis de démontrer l'âge Début Glaciaire (SIM 5.4-5.1) des occupations moustériennes de La Adam et de Ripiceni Izvor ; 
- enfin, la datation IRSL du gisement de Mitoc Valea Izvorului et le réexamen de la séquence lœssique du gisement de Mamaia Sat à la lumière de la chronologie IRSL des séquences lœssiques de référence du SE de la Roumanie (Tuzla, Mostiştea, Mircea Vodă ; Balescu et al., 2003, 2010), nous ont conduits à reconsidérer l'interprétation chronologique de ces deux gisements attribués au Paléolithique moyen. Ces niveaux d'occupation du Paléolithique moyen sont associés au lœss L2 (interstade du SIM 6) à Mitoc Valea Izvorului, au pédocomplexe S2 (SIM 7) et au pédocomplexe de sols rougeâtres vraisemblablement attribué au SIM 9 (S3) à Mamaia Sat. Cette étude nous a ainsi permis de démontrer pour la première fois, l'existence dans l'Est de la Roumanie, d'occupations humaines du Paléolithique moyen attribuables au Pléistocène moyen. Ces conclusions sont parfaitement cohérentes avec les industries lithiques du Paléolithique moyen datées du SIM 6 et du SIM 7 par la méthode IRSL au sein de la séquence lœssique de Velykyi-Glybochok en Ukraine (Lanczont et al., 2014) ainsi qu'avec les industries moustériennes à débitage Levallois de Kozarnika en Bulgarie rapportées au SIM 6 (Guadelli et al., 2005).

Les résultats de notre étude chronologique sont aujourd'hui confortés par la découverte dans les dépôts de grotte de l' «Abri 122 » en Roumanie (Carpates orientales) de niveaux moustériens qui remonteraient au SIM 5, voire un peu avant le SIM 5 (Veres et al., 2017). Le sédiment renfermant le niveau moustérien inférieur a été daté par OSL sur quartz de 106-141 ka et par IRSL sur feldspaths de 99-174 ka.

En Roumanie, les traces d'occupations humaines correspondant à la phase ancienne du Paléolithique moyen, c'est-à-dire d'un âge antérieur au Dernier Interglaciaire (SIM 5.5) étaient jusqu'au début des années 2000 considérées comme inexistantes alors que les industries lithiques du Paléolithique moyen ancien sont nombreuses dans les pays voisins, en Bulgarie à Kozarnika (Guadelli et al., 2005), en Ukraine à Korolevo (Haesaerts et Koulakovka, 2006) ainsi qu'à Velykyi-Glybochok en Podolie (Sytnik, 2000 ; Boguckyj et al., 2009 ; Lanczont et al., 2014). Par contre, de nombreuses industries lithiques du Paléolithique moyen récent (Dernier Glaciaire) ont été mises au jour en Roumanie dans le remplissage de grottes de Transylvanie (Cârciumaru, 1999) et dans des séquences lœssiques à la périphérie des Carpates (Cârciumaru, 1999 ; Păunescu, 1999a et b, 2000). Cette absence d'industrie lithique du Paléolithique moyen en Roumanie avant le Pléistocène supérieur résulte, selon nous, de l'utilisation exclusive de la méthode conventionnelle du ${ }^{14} \mathrm{C}$ au-delà de son champ d'application chronologique $(\sim 40 \mathrm{ka})$ jusqu'à la fin du siècle dernier.

Dans le cadre de cette étude, nous avons démontré la présence de traces d'occupation humaine dès le Pléistocène moyen à la périphérie orientale des Carpates roumaines, le long de la vallée du Danube et de son affluent le Prut, au SIM 6 (épisodes interstadiaires), au SIM 7 et probablement au SIM 9, en parfait accord avec ce que l'on observe au sein des séquences lœssiques de Bulgarie et d'Ukraine.

Ces résultats nous amènent donc à reconsidérer l'étendue chronologique du Paléolithique moyen en Roumanie. Ils remettent en question la chronologie courte du Paléolithique moyen (Păunescu, 1999a, 1999b ; Cârciumaru, 1999) qui s'appuyait uniquement sur des dates ${ }^{14} \mathrm{C}$ et le schéma chronostratigraphique des lœss roumains établi par A. Conea (1969).

En conclusion, le Paléolithique moyen de Roumanie s'inscrit dorénavant dans une chronologie longue comparable à celle observée dans les pays voisins (Ukraine et Bulgarie) et en Europe occidentale où il débute aux environs de 300 ka (Tuffreau, 1979, 1982 ; Bosinski, 1982). Nos travaux ouvrent dès lors de nouvelles perspectives de recherche sur la présence d'occupations humaines au Pléistocène moyen en Roumanie et en Ukraine, le long des vallées du 
Danube et du Dniester qui, au cours du Pléistocène, ont constitué d'importantes routes de migration pour les Hominidés à travers l'Europe.

\section{Remerciements}

Les travaux ont été effectués dans le cadre de la mission archéologique française en Roumanie «Le Paléolithique de Roumanie », ministere français des Affaires étrangères.

Le spectromètre du Département Homme et Environnement du Muséum national d'Histoire naturelle, utilisé dans cette étude, a été acquis grâce à l'aide de la région Île-de-France.

\section{Références}

Adamiec, G., Aitken, M., 1998. Dose-rate conversion factor: update. Ancient TL 16, 37-50.

Alexandrescu, E., Balescu, S., Tuffreau, A., 2004. Nouvelles données chronologiques, technologiques et typologiques sur le Paléolithique supérieur ancien de la Plaine roumaine du Danube : le gisement de Giurgiu-Malu Roşu. Anthropologie 108, 407-423

Auguste, P., 2009. Évolution des peuplements mammaliens en Europe du Nord-Ouest durant le Pléistocène moyen et supérieur. Le cas de la France septentrionale. Quaternaire 20 (4) 527-550.

Auguste, P., 2012. L'homme et l'animal au Pléistocène en France septentrionale. Un quart de siècle de recherches paléontologiques et archéozoologiques dans le nord de la France. Habilitation à diriger des recherches. Université de Lille 1 (2 volumes, 251 p.).

Bahain, J.-J., Laurent, M., Falguères, C., Voinchet, P., Farkh, S., Tissoux, H., 2002. Datation par résonance paramagnétique électronique (RPE) des formations fluviatiles pléistocènes et des gisements archéologiques ou paléontologiques associés. Quaternaire 13, 91-103.

Bahain, J.J., Yokoyama, Y., Falguères, C., Sarcia, M.N., 1992. ESR dating of tooth enamel: a comparison with K-Ar dating. Quater. Sci. Rev. 11, 245-250.

Balescu, S., 2013. Datation IRSL des lœss de Dobrogea ; implications pour la chronologie des occupations paléolithiques. In: Saint-Martin, J. (Ed.), Recherches croisées en Dobrogea Editura Amanda Edit. Bucarest, pp. 87-95.

Balescu, S., Lamothe, M., Mercier, N., Huot, S., Balteanu, D., Billard, A., Hus, J., 2003. Luminescence chronology of Pleistocene lœss deposits from Romania: testing methods of age correction for anomalous fading in alkali feldspars. Quater. Sci. Rev. 22, 967-973.

Balescu, S., Lamothe, M., Panaiotu, C.G., Panaiotu, C.E., 2010. La chronologie IRSL des séquences lœssiques de l'Est de la Roumanie. Quaternaire 21, 115-126.

Balescu, S., Tuffreau, A., Dobrescu, R., 2015. Mamaia Sat (Romania): a Late Middle Pleistocene Palaeolithic site? Anthropolog. Brno LIII 1-2, 157-166.

Boguckyj, A.B., Lanczont, M., Lacka, B., Madeyska, T., Sytnyk, O., 2009. Age and palaeoenvironment of West Ukrainian Paleolithic: the case of Velykyi Glybochok multi-cultural site. J. Archaeol. Sci. 36, 1376-1389.

Bosinski, G., 1967. Die mittelpaläolitischen Funde im westlichen Mitteleuropa. Fundamenta, Reihe A4, Köln und Graz.

Bosinski, G., 1982. The Transition Lower/Middle Palaeolithic in Northwestern Germany. In: Ronen, A. (Ed.), The transition from Lower to Middle Palaeolithic and the Origin of Modern Man. BAR Int. Series, 151. pp. $165-173$.

Bosinski, G., 2000-2001. Paleoltico medio en Europa central. Zephyrus 53-54, 79-142.

Boudadi-Maligne, M., 2010. Les Canis pléistocènes du sud de la France : approche biosystématique, évolutive et biochronologique. (Thèse de Doctorat) Université de Bordeaux 1 (450p.).

Brennan, B.J., Rink, W.J., Mcguirl, E.L., Schwarcz, H.P., Prestwich, W.V., 1997. Beta doses in tooth enamel by "One Group" theory and the Rosy ESR dating software. Radiat. Measur. 27, 307-314.

Buggle, B., Hambach, U., Glaser, B., Gerasimenko, N., Marković, S., Glaser, I., Zöller, L., 2009. Stratigraphy, and spatial and temporal paleoclimatic trends in Southeastern/Eastern loess-paleosol sequences. Quater. Inter. 196, 86-106.

Cârciumaru, M., 1999. Le Paléolithique en Roumanie. Editions Jérôme Million, Grenoble.

Chabai, V., Marks, A., Konigal, K., 2004. Crimea in the context of the eastern Middle Paleolithic and early Upper Paleolithic. In: Chabai, V., Monigal, K., Marks, A. (Eds.), The Middle and Early Upper Paleolithic of eastern Crimea (The Paleolithic of Crimea III). ERAUL, 104, pp. 419-440.

Chirica, V., 2001. Gisements paléolithiques de Mitoc. Le Paléolithique supérieur de la Roumanie à la lumière des découvertes de Mitoc. Helios, Iaşi.

Pour citer cet article : Balescu, S.., et al., Nouvelles données sur la chronologie des sites paléolithiques en contexte lœssique du Nord-Est et du Sud-Est de la Roumanie (Périphérie orientale des Carpates). L'Anthropologie (2018), https://doi.org/10.1016/j.anthro.2018.02.001 
Conea, A., 1969. Profils de lœss en Roumanie. In: Fink, J (Ed.), La stratigraphie des lœss d'Europe. Supplément du Bulletin de l'Association Française pour l'Étude du Quaternaire. INQUA, pp. 127-134.

Constantin, D., Begy, R., Vasiliniuc, S., Panaiotu, C., Necula, C., Codrea, V., Timar-Gabor, A., 2014. High-resolution OSL dating of the Costinesti section (Dobrogea, SE Romania) using fine and coarse quartz. Quater. Inter. 334-335, 20-29.

Cosac, M., Muratoreanu, G., Veres, D., Nja, L., Schmidt, C., Hambach, U., Alexandru, R., Cuculici, R., Buzea, D.L., Margarit, M., Dumitracu, V., Vasile, S., Petculescu, A., Denes, I., 2017. Multi-proxy archaeological investigations of a Middle Paleolithic occupation context in Eastern Transylvania, Romania. Quater. Int., http://dx.doi.org/10.1016/ j.quaint.2017.09.014 (In press).

Doboş, A., Trinkaus, E., 2012. A new AMS radiocarbon date for Middle Paleolithic Layer 4 of Ripiceni-Izvor, Romania. Materiale şi Cercetări Arheologice (serie nouă) VIII, 7-10.

Fitzsimmons, K.E., Marković, S.B., Hambach, U., 2012. Pleistocene environmental dynamics recorded in the loess of the middle and lower Danube basin. Quater. Sci. Rev. 41, 104-118.

Germonpré, M., 1993. Osteometric data on Late Pleistocene mammals from the Flemish Valley, Belgium. Documents de travail de 1'Institut Royal des Sciences naturelles de Belgique. 72 (135 p.).

Grün, R., 1989. Electron spin resonance (ESR) dating. Quater. Inter. 1, 65-109.

Grün, R., Schwarcz, H.P., Chadam, J.M., 1988. ESR dating of tooth enamel: coupled correction for U-uptake and U-series disequilibrium. Radiat. Meas. 14, 237-241.

Grün, R., Katzenberger-Apel, O., 1994. An alpha irradiator for ESR dating. Ancient TL, 12. pp. 35-38.

Guadelli, J.-L., Delpech, F., 2000. Les grands mammifères du début du Paléolithique supérieur à Temnata. In: Ginter, B., Kozlowski, J.K., Guadelli, J.-L., Laville, H. (Eds.), Temnata Cave. Excavations in Karlukovo Karst Area, Bulgaria, 2. Jagellonian University Press, Cracow (part.1., 90 p.).

Guadelli, J.L., Sirakov, N., Ivanova, S.T., Sirakova, S.T., Anastassova, A.E., Courtaud, P., Dimitrova, I., Djabarska, N., Fernandez, P.H., Ferrie, R.C., Fontugne, M., Gambier, D., Guadelli, A., Iordanova, D., Iordanova, N., Kovatcheva, M., Krumov, I., Leblanc, J., Mallye, C.L., Marinska, J.B., Miteva, M., Popov, V., Spassov, V., Taneva, R., TisteratLaborde, S.T., Tsanova, N.S.T., 2005. Une séquence du Paléolithique inférieur au Paléolithique moyen récent dans les Balkans ; la grotte Kozarnika à Orechets (Nord-Ouest de la Bulgarie). In: Molines, N., Moncel, M.H., Monnier, J.L. (Eds.), Les premiers peuplements en Europe. BAR Int. Series, 1364. pp. 87-103.

Haesaerts, P., 2007. Mitoc-Malu Galb cadre stratigraphique et chronologique. In: Otte, M., Chirica, V., Haesaerts, P. (Eds.), L'Aurignacien et le Gravettien de Mitoc-Malu Galben (Moldavie Roumaine), 72. ERAUL, Liège, pp. $15-41$.

Haesaerts, P., Koulakovka, L., 2006. La séquence pédosédimentaire de Korolevo (Ukraine transcarpatique) : contexte pédosédimentaire et chronologique. In: Koulakovska, L. (Ed.), The European Middle Palaeolithic. Institute of Archaeology of the National Academy of Sciences of Ukraine, Kiev, pp. 21-37.

Huntley, D.J., Lamothe, M., 2001. Ubiquity of anomalous fading in K-feldspars, and the measurement and correction for it in optical dating. Can. J. Earth Sci. 38, 1093-1106.

Hütt, G., Jaek, I., Chonka, J., 1988. Optical dating: K-feldspars optical response stimulation spectra. Quater. Sci. Rev. 7, 381-385.

Ivanovich, M., Harmon, R.S., 1992. Uranium-series disequilibrium: applications to earth, marine and environmental sciences, 2nd Edition. Clarendon Press, Oxford.

Jöris, O., 2006. Bifacially backed knives (Keilmesser) in the Central European Middle Palaeolithic Axe Age. In: GorenInbar, N., Sharon, G. (Eds.), Acheulian tool-making from quarry to discard. Equinox Publishing Ltd, London Oakville, pp. 287-310.

Kolfschoten, T.V., 1990. The evolution of the mammal fauna in The Netherlands and the middle Rhine Area (Western Germany) during the late Middle Pleistocene. Meded. Rijks Geol. Dienst 4, 1-69.

Kolfschoten, T.V., 1992. Aspects of the migration of mammals to Northwestern Europe during the Pleistocene, in particular the reimmigration of Arvicola terrestris. Courier Forsch. Inst. Senckenberg. 153, 213-220.

Lanczont, M., Madeyska, T., Bogucki, A., Sytnyk, O., Kusiak, J., Frankowski, Z., Komar, M., Nawrocki, J., Zogala, B., 2014. Stratigraphic position and natural environment of the oldest Middle Paleolithic in central Podolia, Ukraine: New data from the Velykyi Glybochok site. Quater. Int. 326-327, 191-212.

Louguet-Lefèvre, S., 2005. Les mégaherbivores (Éléphantidés et Rhinocérotidés) au Paléolithique moyen en Europe du Nord-Ouest. Paléoécologie, taphonomie et aspects palethnographiques. Archaeopress, Oxford. Br. Archaeol. Rec. Int. Series 1451, 1-267.

Markova, A.K., 2007. Pleistocene mammal faunas of Eastern Europe. Quater. Inter. 160, 100-111.

Mejdahl, V., 1988. Long-term stability of the TL signal in alkali feldspars. Quater. Sci. Rev. 7, 357-360.

Mejdahl, V., 1989. How far back: life times estimated from studies of feldspars of infinite ages. In: Aitken, M.J. (Ed.), Synopses from a Workshop on "Long and Short Range Limits in Luminescence Dating". The Research 
Laboratory for Archaeology and the History of Art, Oxford University, Oxford, (Occasional Publication 9), pp. 53-58.

Otte, M., Chirica, V., 1993. Atelier aurignacien à Mitoc-Malu Galben. Préhistoire européenne, 3. pp. $55-67$.

Otte, M., Chirica, V., Haesaerts, P., 2007. L'Aurignacien et le Gravettien de Mitoc Malu Galben (Moldavie roumaine). ERAUL 72, Liège.

Panaiotu, C.G., Panaiotu, E.C., Grama, A., Necula, C., 2001. Paleoclimatic record from a Lœss-Paleosol Profile in Southeastern Romania. Phys. Chem. Earth (A) 26, 893-898.

Păunescu, A., 1993. Ripiceni-Izvor. In: Palaeolithic şi Mezolitic. Studiu monografic. Editura Academiei Române, Bucureşti.

Păunescu, A., 1999a. Paleoliticul şi Epipaleoliticul de pe teritoriul Moldovei cuprins între Carpaţi şi Siret, I. ed. Satya Sai, Bucureşti.

Păunescu, A., 1999b. Paleoliticul şi Mezoliticul de pe teritoriul Dobrodgei, II. ed. Satya Sai, Bucureşti.

Păunescu, A., 2000. Paleoliticul şi Mezoliticul din spaţiul cuprins între Carpaţi şi Dunăre. Editura AGIR, Bucureşti.

Petculescu, A., 2003. New data on the evolution of the Arvicola (Rodentia, Mammalia) from the karst of Romania. Theor. Appl. Karstol. 16, 99-103.

Petculescu, A., Samson, P.-M., 2001. Aspecte climatice ale ultimuliu ciclu glaciar, bazate pe asociatiile de micromamifere din carstul Dobrogei. In: Ecocarst 2.

Prescott, J.R., Hutton, J.T., 1994. Cosmic ray contributions to dose rates for luminescence and ESR dating: large depths and long-term time variations. Radiat. Meas. 23, 497-500.

Radan, S.C., 2012. Towards a synopsis of dating the loess from the Romanian Plain and Dobrogea: authors and methods through time. Geo. Eco. Marina 153-172.

Radulescu, C., Samson, P.M., 1962. Sur un centre de domestication du Mouton dans la grotte La Adam en Dobroudja. Z. f. Tierzücht. u. Züchtungsbiol. 76, 282-320.

Röttger, U., 1987. Schmelzbandbreiten and Molaren von Schermäusen (Arvicola Lacépéde, 1799). Bonner Zoologische Beitraege 38, 95-105.

Samson, P.M., 1971. La stratigraphie du Quaternaire supérieur en Roumanie. In: Études sur le Quaternaire dans le Monde, VIII ${ }^{\mathrm{e}}$ Cong. INQUA, Paris, 1969. pp. 629-636.

Shao, Q., Bahain, J.-J., Falguères, C., Peretto, C., Arzarello, M., Minelli, A., Thun Hohenstein, U., Dolo, J.-M., Garcia, T., 2011. New ESR/U-series data for the early Middle Pleistocene site of Isernia La Pineta, Italy. Radiat. Meas. 46, 847-852.

Shao, Q., Bahain, J.-J., Dolo, J.-M., Falguères, C., 2014. Monte Carlo approach to calculate US-ESR ages and their uncertainties. Quater. Geochronol. 22, 99-106.

Sytnik, O., 2000. СереднійпалеолітПоділля. (Middle Paleolithic of Podillia).National Ukranian Academy of Science, Lviv.

Timar, A., Vandenberghe, D., Panaiotu, E.C., Panaiotu, C.G., Necula, C., Cosma, C., van den Haute, P., 2010. Optical dating of Romanian lœss using fine-grained quartz. Quater. Geochronol. 5, 143-148.

Timar-Gabor, A., Vandenberghe, D.A.G., Vasiliniuc, S., Panaiotu, C.E., Panaiotu, C.G., Dimofte, D., Cosma, C., 2011. Optical dating of Romanian loss: a comparison between silt-sized and sand-sized quartz. Quater. Int. 240, 62-70.

Tuffreau, A., 1979. Les débuts du Paléolithique moyen dans la France septentrionale. Bull. Soc. Prehistor. Fr. 76, $140-142$.

Tuffreau, A., 1982. The transition from Lower to Middle Paleolithic in Northern France. In: Ronen, A. (Ed.), The transition from Lower to Middle Paleolithic and the origin of Modern Man. Bar Int. Series, 151. pp. 137-149.

Tuffreau, A., Chirica, V., Balescu, S., Haesaerts, P., 2009. Nouvelles recherches sur le gisement paléolithique de Mitoc Valea Izvorului (département de Botosani), Fouilles 2003-2004. Materiale şi cercetari arheologice (serie noua) V, 21-31.

Tuffreau, A., Dobrescu, R., Petculescu, A., Stiucă, E., Balescu, S., Lanoe, F., Wismer, M., 2013. La grotte La Adam : un repaire de carnivores visité par les chasseurs du Paléolithique. In: Saint-Martin, J. (Ed.), Recherches croisées en Dobrogea, Editura Amanda Edit, Bucarest. pp. 75-86.

Tuffreau, A., Dobrescu, R., Balescu, S., Ciornei, A., 2014. Occupations du Paléolithique supérieur ancien dans la Plaine roumaine du Danube à Vădastra-Măgura Fetelor et à Ciuperceni-La Vii 1 : industries lithiques, matières premières et déplacements. In: Otte, M., Lebrun-Ricalens, F. (Eds.), Modes de contacts et de déplacements au Paléolithique eurasiatique". ERAUL 140 et Archéologiques. pp. 279-302.

Valoch, K., 1968. Gisement du Pléistocène supérieur à Mamaia sur la côte de la Mer Noire. In: La Préhistoire, problèmes et tendances. Centre national de la recherche scientifique, Paris465-472.

Valoch, K., 1993. Les industries du Paléolithique moyen de Mamaia-Sat, Roumanie. Anthropologie 97, $239-264$. 
Vasiliniuc, S., Vandenberghe, D.A.G., Timar-Gabor, A., Panaiotu, C., Cosma, C., van den Haute, P., 2012. Testing the potential of elevated temperature post-IR IRSL signals for dating Romanian loess. Quater. Geochronol. 10, 75-80.

Veres, D., Cosac, M., Schmidt, C., Muratoreanu, G., Hambach, U., Hubay, K., Wulf, S., Karatson, D., 2017. New chronological constraints for Middle Palaeolithic (MIS 6/5-3) cave sequences in Eastern Transylvania. Romania. Quater. Int., http://dx.doi.org/10.1016/j.quaint.2017.07.015 (In press). 